\title{
Comportement et stabilité des régulateurs contenant des éléments à caractéristique non linéaire
}

\author{
(Précédé d'un aperçu de la méthode de Nyquist et des méthodes dérivées \\ appliquées à l'hydraulique.)
}

\section{Behaviour and stability of govenors with elements of non-linear characteristics}

(Preceeded by an outline of the Nyquist method and methods derived from it applied to hydraulics.)

PAR R. MLYER

INGÉNIEUR AUX ÉTABIISSEMENTS NEYRPIC (GRENOBLE)

\begin{abstract}
Analyse des installations de régulation et définilion des termes employés. Théories el méthodes d'étude des régulateurs à élements linéaires.

Ceci constitue une introduction ì l'étude du comportement des installations antomatiques en hydratulique.

On se contente habituellement d'ótudier des iquations différentielles linéaires. Effectivement, less systimes linéaires constituent, dans certains cas, une première approximalion que l'on a alors intérêt a examiner en premier lieu à cause de sa grande simplicité et du fait qu'elle est parfois suffisante.

Si cette approximation est impossible ou si elle s'avère insuffisante, il convient de revenir anx bases physiques, afin de prendre en considération ce que la linéarisation conduit à négliger. L'auteur commence par l'étude, à partir de lent base physique, des systèmes dans le domaine linéaire. Cette étude, déjà intéressante en elleméme, sert d'introduction à ane étude beaucoup plus générale qui doit être publiée nltérieurement.
\end{abstract}

Governors and governing study methods. Anal$y$ sis of governing installations and definition of terms used. Theories and sludy methods of linear element governors.

This forms an introduction to the stady of the behaviour of automatic, hydraulic installations. It is usual to study but the differential linear equations. In some cases, linear systems do indeed form a first approximation which it is wise to ertamine from the start, given its great simplicity and the fact that it is sometimes sufficient.

If it is not possible to make this approximation, or if it proves to be insufficienl, the physical bases should be reverted to, in order to take into consideration that which linearization excludes.

The anthors finst studies the linear type systems, starting from their physical bases.

This stady, which is itself of great interest, serves as an introduction to a far more general investigation to be published at a later date.

\section{$S O M M A I R E$}

\section{I. - INTroduction.}

II. - Les RÉgulateuns et les MÉThodes D'ÉtUde DE LA RÉGULATION.

II-1. - Ce qu'on demande à un régulateur.

II-2. - Les installations automatiques et les méthodes d'étude de la régulation.

II-3. - Avantages spécifiques de la méthode de Nyqurst et des méthodes dérivées.
II-4. - Cas où les méthodes de HurwitzRou'tr sont intéressantes.

III. - DESCRIPTION DES INSTALLATIONS DE RÉgULATION ET DEFINITION DES TERMES EMPLOYÉS.

III-1. - Description de deux installations industrielles simples.

III-2. - Les différents régulateurs employés.

III-3. - Décomposition des éléments des boucles de régulation. 
111-4. - Les quadripôles généralisés.

III-5. - Etude des éléments des boucles de régulation.

III-6. - Les comparateurs (ou discriminatours).

IV. 'THÉRIES l' METHODES D'EYUDE DES INSTALLATIONS AUTOMATIOUES A BLEMENTS IINEAIRES.

IV-1. Méthodes d'Hunwrtz-RouTh, LFoNHARD, etc...

IV-2.-- Méthode de Nroust

IV-3. Systemes a minimun de phase.

IV-4. - Les installations automatiques en tant qu'integrateurs.

(Seals, res qualre premiers chapilres sonl publiés dans le present naméro.)

Y. ETUDI: DES INSTALATIONS A ÉLEMENTS NON InEAIRES. EXTENSION DU CRITERE DE NYOUIST. LAMTES DE L'DXTENSION.

V-1. L'étude se linite aux installations autom matiques infustriclles.

v-2. Description des installations non li- néaires et légitimation de la méthode employse.

V-3. - Difrérence entre éléments linéaires et non linéaires.

V-4. - Stabilité des cycles limites.

V-5. - Emploi pratique de la méthode.

\section{VI. - EXEMPLES.}

VI-1. - Cas élémentaires où les résultats sont connus par méthode directe.

-- Régulateur direct de niveau avec jeu et adduction par une conduite courte.

- Régulateur a tout ou rien avec adduction a contps de bélier de masse.

VI-2. - Cas solubles uniquement par la méthode de Nyoust généralisée.

- Régulateur par tout ou rien el coups de bélier d'onde dans l'adduction.

- Régulateur par tout ou rien et cheminée d'équilibre.

- Régulateur à vilesse proportionnelle à l'écart avec jeu et installation avec coup de bélier.

VII. - RÉsumé Finat ete conclusrons.

\section{I. - INTRODUCTION}

Depuis les annees dapres-guerre, le problème de la régulation a fait l'objet de nombreuses pubiscations et tout particulierement dans la Homlle Blanche qui a facilité le dialogue entre les spécialistes en la matiere. Ces derniers avaient surtout en vue de préciser les notions de stabilité d'un régulateur aulomatique de vitesse d'une turbine hydraulique el de delinir les conditions oplimum de réglage de la fréquence des réseaux de distribution électrique. Mais en hydraulique la régulation ne se limite pas a ce simple cas, et c'est là l'excuse que nous alléguerons pour oser revenir sur cette question. En effet, le récent développement des grands réseaux d'irrigation, où il convient de régler débits et niveaux, a conduit les techniciens à concevoir des appareils automatiques (vannes, obturateurs, etc.) qui, placés dans l'ensemble maillé d'une surface irriguée, doivent coordonner leur fonctionnement et contribuer ainsi à la stabilité de marche du réseau. De même encore les Laboratoires d'Hydraulique. par nécessité d'une précision toujours plus grande dans les mesures, font très souvent appel à des systèmes à commande indirecte asservie, que peuvent soulever des problèmes de régulation fort complexes.

Aussi, pour la commodité de notre discours, nous désignerons les différents appareils auxquels nous venons de faire allusion (régulateur de turbine, vannes et obturateurs automatiques, elc.) sous le vocable général de "régulateurs hydrau- liques ». Cependant, que l'on ne s'y trompe pas, celte dénomination unique rappelant le but commun de ces appareils (réglage d'une grandeur physique) cache la grande diversité de leur structure et si les régulateurs de turbines hydrauliques évoquent à l'esprit des lecteurs un dispositif suffisamment bien défini, les systimes mis en jeu aussi bien pour l'irrigation que pour les mesures restent trop divers pour les résumer par un schéma de principe unique; corrélativement on conçoit donc que leur régulation soit souvent fort complexe et surtout constitue chacue fois un cas particulier à examiner attentivement.

Aussi, pour mettre de l'ordre dans notre étude, scrons-nous amenés à classer ces systèmes en tenant compte des difficultés plus ou moins grandes que soulève théoriquement leur régulation.

Nous distinguerons donc essentiellement les systèmes " linéaires » et les systèmes «non linćaires » dont les définitions seront précisées dans le corps de notre article. Dans l'état actuel de la théorie, pour les premiers seuls il est possible de déterminer d'une manière précise les relations auxquelles ils doivent satisfaire dans les divers cas envisagés. Plusieurs méthodes sont utilisées; les unes purement mathématiques, comme celle d'HuRwiTz-Routh, permettent d'obtenir un résultat général de forme simple, extrêmement précieux pour contrôler rapidement la stabilité d'une installation; cet avantage a rendu d'ailleurs courante leur utilisation. D'autres, 
apparentées à la méthode de NyQuis'T, font appel à des considérations graphiques; elles ont été jusqu'à présent moins connues des ingénieurs hydrauliciens, mais nous pensons que l'avenir leur rendra justice car elles sont susceptibles d'extension et par là peuvent devenir un guide fort utile pour l'étude des problemes « non linéaires $\gg$.

L'une de ces extensions constitue l'objet principal de notre exposé; nous avons en effet profité des particularités spéciales des systèmes de régulation industrielle pour calculer leur stabilité et éventuellement leur cycle limite de pompage. Ces particularités sont évidemment des restrictions du point de vue des systemes phy- siques en général, mais non du point de vue des régulateurs hydrauliques qui seuls nous intéressent. Certes, nous serons conduits à consentir également certaines approximations, mais nous ne le ferons qu'après les avoir justifices pour tous les problemes que nous avons en vue.

Chemin faisant, nous ferons quelques digressions pour rappeler certaines définitions et comparer les méthodes entre elles; que l'on nous pardonne ees redites, mais il nons a paru plus agréable de hien préciser les termes employés et les théories utilisées afin d'éviter tout malentendu entre nous et de ne pas lasser l'attention de nos lecteurs par un texte trop concis et par la trop elliptique.

\section{II. - LES RÉGULATEURS ET LES MÉTHOdES D'ÉTUDE DE LA RÉGULATION}

\section{II-1. - Ce qu'on demande à un régulateur.}

Un régulateur est employé pour régler une grandeur à une valeur donnée à l'avance, en agissant sur le systeme à régler.

Exemple : dans un modèle réduit, on se propose de reproduire une loi de marce : hauteur d'eau en fonction du temps : $h=h(t)$. On peut agir sur le débit entrant dans le modèle (fig. 1 ).

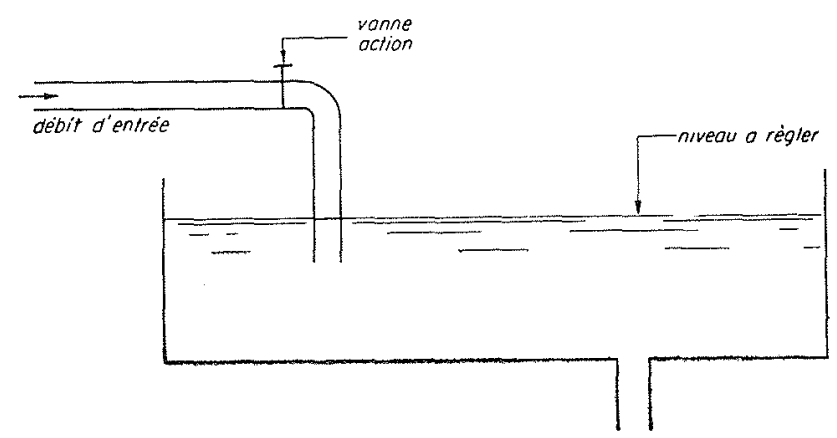

Fir. 1. - Soluéma (simplifié à l'extròme) d'une installation à niveau réglé.

Souvent le problème consiste à maintenir une grandeur à une valeur constante.

Exemple : la fréquence d'un réseau électrique.

Malheureusement, il n'existe pas de régulateur permettant d'obtenir un réglage parfait : le régulateur n'agit que s'il détecte nne erreur de réglage; sans erreur, pas d'action possible du régulateur. D'ailleurs, très souvent, on ne recherche aucunement un réglage parfait (surtout en hydraulique). Dans une installation hydro-électrique, ou dans une installation d'adduction d'eau, un réglage parfait provoquerait des coups de bélier funestes dans les conduites et galeries d'amenée d'eau. Bien d'autres causes peuvent rendre désirable la limitation de la précision; ainsi, un correcteur de niveau pour modèle réduit (voir fig. 1) ne doit pas « essayer de corriger » les fluctuations turbulentes ou les rides et vagues superficielles dues à l'agitation désordonnée du plan d'eau (on verra plus loin ce qu'on attend de lui).

La question centrale de la régulation est donc la précision. Or, la précision n'est pas une propriété bien définie et ne peut pas être caractérisée dans tous les cas par une seule grandeur. Nous allons roir que, selon l'emploi du régulateur, différentes définitions peuvent ètre adoptées.

Ainsi, pour le correcteur de niveau dont il a été question plus haut, on peut définir une précision en fonction de la fréquence. Une perturbation sinusoïdale établie en régime permanent : A $\cos \omega t$, ne doit pas donner naissance à une erreur plus grande que $=\mathrm{A} \cos (\omega t+\varphi)$. Dans le cas qui nous intéresse, $\approx(\omega)$ doit avoir une allure comparable à la figure 2.

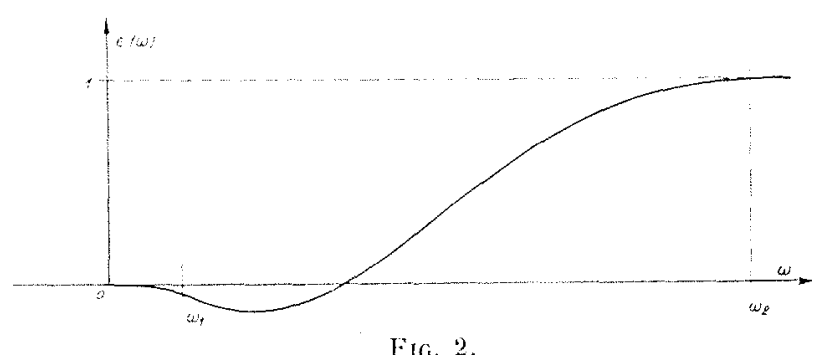

Si $\omega \rightarrow 0$, il faut que $\varepsilon \rightarrow 0$ pour que le niveau moyen soit correct. De plus, la marée dont la période fondamentale est $\omega_{1}$ doit ètre reproduite correctement (avec, par exemple, 5 premières 
hammoniques). d la fréquence $(1)=\omega_{2}$ qui correspond à l'ordre de grandeur de la fréquence d'agitation générale du modèle, le régulateur ne doit plus répondre : l'erreur est égale à la perturbation.

On peut définir bien d'autres grandeurs caractérisant la précision. Par exemple, on peut essayer de minimiser (de différentes manières d'ailleurs) l'erreur que commet le système s'il est soumis à une perturbation accidentelle de forme donnée.

La figure 3 en montre un exemple.
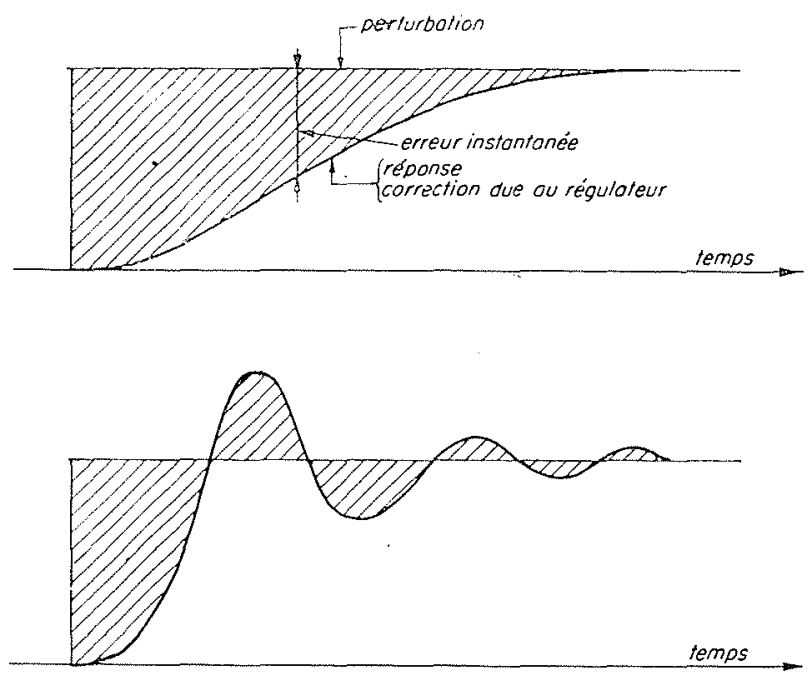

Fic. 3 .

On peut chercher a minimiser la surface hachuré et déftuir un temps de réponse : « aire hachurée » divisée par « hateur de la perturbation».

Pour de plus amples discussions à ce sujet, on peut voir les références ${ }^{1}:$ [21] [34] [39] [41] 42] [49] [52] [54].

Bien que la précision ne soit pas définie d'une façon unique, on peut quand même classer les installations automatiques, en hydraulique, grosso modo de la façon suivante, en ce qui concerne la précision demandée :

Très grande précision : appareils de réglage de modeles réduits et appareils de mesures.

Bonne précision moyenne : réglage fréquencepuissance des réseaux électriques.

Précision plus faible : appareillages automatiques d'irrigation et adduction d'eau.

Néanmoins, il existe un critère toujours nécessaire pour qu'on puisse parler de précision (quelle

1. Les chiffres entre crochets renvoient it la bibliographie à la fin de l'article. que soit la définition qu'on en donne) : il faut que l'installation automatique envisagée soit suffisamment stable. Ceci veut dire qu'à l'intérieur du champ de fonctionnement du système l'erreur en régime établi doit être inféricure à une valeur donnée jugée admissible?

La stabilité n'est qu'un cas particulier de la précision : c'est la précision quand depuis longtemps il n'y a plus eu de perturbations (voir fig. 4) :

Si « $a »$ est suffisamment petit, on dit que la stabilité est suffisante.

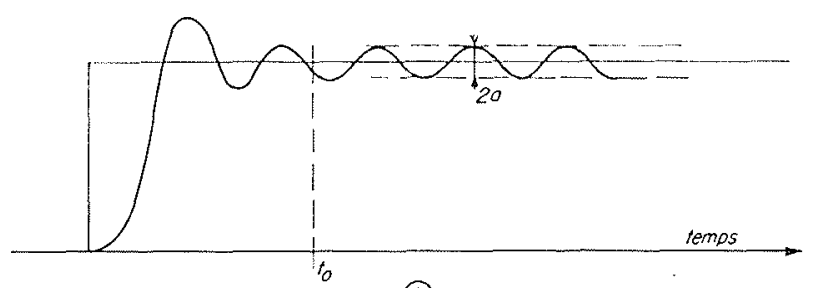

(A)

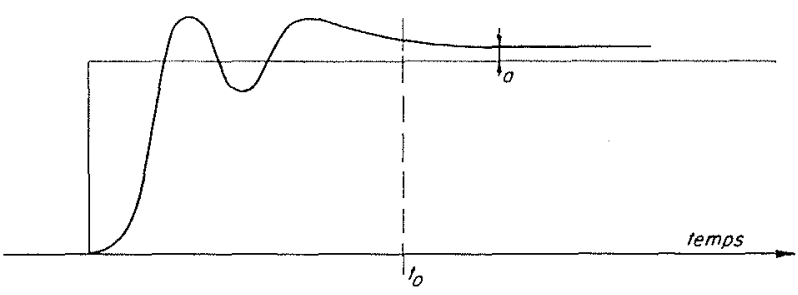

(B)

FIG. 4.

A partir du temps $t_{0}$ après une perturbation (échelon unite) le système considéré en $4 \mathrm{~A}$ oscille avec une amplitude pratiquement constante et égale $a \ll a »$, celui en $4 \mathrm{~B}$ a une erreur constante égale à $《 a \gg$.

II est à remarquer, que jusqu'à présent, à peu près tous les auteurs définissent la stabilité par $a=0$. Ceci provient du fait qu'ils n'étudient que les systèmes dits «linéaires » (voir définition plus loin) et revient à étudier, en première approximation (linéarisation) certaines installations. Il est clair que, d'une façon générale, on n'a pas besoin d'annuler complètement « $a$ » (voir, par exemple, [52] - linéarisation par balayage). D'ailleurs, il n'est jamais possible d'annuler $« a »$ en toute rigueur.

En dehors de la stabilité qui est caractérisée par « $a »$, on cherche souvent la loi suivant laquelle le système perturbé tend vers son régime d'équilibre, loi permettant de définir ce que l'on appelle le degré de stabilité [52]. Cette loi n'est pas unique (sauf pour les systèmes linéaires).

2. On sait que pour les systèmes linéaires (les seuls étudiés jusqu'ici), la stabilité exige entre autre la « constance dans le temps $\Rightarrow$ pour $t \rightarrow \infty$. On est obligé, ici, d'clargir le sens. 
Elle est lice à la précision dynamique qui, elle non plus, n'est pas susceptible d'une définition unique.

Pour qu'une installation automatique soil acceptable, il faut qu'elle tende très rapidement vers le régime établi; néanmoins, d'autres considérations de précision peuvent intervenir [40].

Dans cet article, nous allons surtout nous intéresser à la stabilité et à la précision des installations automatiques.

Toutefois, il convient de ne pas perdre de vue que les régulateurs ont souvent des fonctions annexes tres importantes, par cxemple la limitation des coups de bélier dans les conduites forcées et galeries des installations hydro-électriques ou d'adduction d'eau (et d'une facon plus générale l'actionnement de divers dispositifs de sécurité).

\section{II-2. - Les installations automatiques et les méthodes d'étude de la régulation.}

Les inslallations automatiques se composent d'une facon générale de l'installation à régler (par exemple ce qui est représenté sur la figure 1) et du régulateur [53]. Dans la littérature allemande, on trouve les termes «Regelstrecke» et «Regler». Le régulateur est donc un appareil qui sert à régler au mieux une installation donnéc.

Selon la complexité du système à régler et la précision demandée, le régulateur sera plus ou moins compliqué et la méthode d'étude de la régulation devra être plus ou moins puissante.

A notre connaissance, les méthodes suivantes ont été employées en hydraulique :

1. Méthode directe, appliquée dans [45] [50].

2. Methode d'Hurwitz-Routh el ses généralisaltions, décrites dans [21] [33] [42] [44] [45] [46] [47], appliquées dans [1] [2] [3] [4] [5] [22] [24] [31 $[35]$ [58].

3. Méthode de Léonmari) et ses généralisations décrite dans [42] [47], appliquée dans [42] et a cours de plusicurs études NFrnPIC non publiées.

4. Méthode de Nroust appliquée dans 5$][16$ [18] [19] [21] [29] [32].

Une méthode intéressante a été mise au point par M. Nisse [53]; elle met en évidence la division du système automatique 'n systeme à régier of régulateur.

De plus, il existe différentes méthodes dérivées de celle de Nrovist et différentes améliorattions qui sont employées par les électriciens [52]. Nous les déerirons plus loin. Il existe également d'autres méthodes moins connues comme celles dites « des séparatrices» $[38]$.

Toutes ces méthodes, saut les méthodes direcles, s'appliquaient jusqu'à présent uniquement aux systèmes dits « linéaires ». Une partie des systèmes hydrauliques peuvent ètre considérés conme tels en toute première approximation.

En ce qui concerne les systèmes non linéaires seuls quelques cas particuliers très élémentaires (et par conséquent n'ayant pas de grandes applications pratiques) ont pu être étudiés jusqu'à ces derniers temps [51] [59). Récemment, une méthode plus puissante a été mise au point (sé-

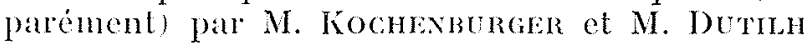
[27] [36]. Nous nous proposons de décrire et de justifier une méthode plus générale permettant de traiter tous les cas que nous connaissons:-

La comparaison des différentes inéthodes s'appliquant aux systèmes linéares est très délicate. M. Cúvon écrit [10] en parlant de "cerlaines méthodes nouvelles » (il parle de la méthode Nyouist) :

«1. Elle permettent de traiter des cas où la méthode chassique d'Hurwitz conduirait a des calculs inextricables, sinon impossibles à effectuer, en remplacant les développements mathématiques par une construction graphique ou semi-graphique.

«2. Elles permettent de se faire une représentation très eoncrète des phénomènes dynamiques intervenant dans les problemes de réglage.

«3. Elles facilitent Ie calcul des variations de la grandeur à régler résultant d'une perturbation du dispositif de réglage.

Il est à remarquer que M. Cóvon ne se serl pratiquement pas des dernicres améliorations de la méthode de Nrousto (il est vrai que la régulation des turbines en fréquence-puissance fait toujours intervenir des systemes " non a minimum de phase " ", néanmoins, on peut souvent lacilement tenir compte d'un déphaseur pur connu).

Persomellement, nous pensons que la méthode de Nroust est, dans beaucoup de cas, la meil-

1. Physiquement, cette methode se confond tres tapj. dement avee celle de Nyquist : on teste le systeme avec des excitations sinusoidales. Elle ne présente néanmoins pas tous les avantages physiques de cette derniere: par contre. il semblerait qu'elle se prete micux à cerlains calculs.

2. Nous avons applis recemment, au cours d'une conference que M. LoEs a tenue a Grenoble le 25 arril 1951, que celui-ci al fait des recherehes dans la meme direction fous avons aussi appris par la suite qu'il a publie nu article dans les Annales des Teleommanicafions de december 1951.

A.D.L.R. - Ce manuserit de M. Mrum nous a be remi. (*) octobre 1951.

3. Yoir plas loin la definition. 
leure. Elle est, jusqu'à présent, la seule applicable atux systèmes non linéaires. Néammoins, pour les systemes simples ef pour une premiere étude (linéarisation), la méthode d'Hen wiz-RovTn est souvent aussi bonne (ou mème meilleure). Cela dépend essentiellement de l'entrainement de l'opérateur.

\section{II-3. - Avantages spécifiques de la méthode de Nyquist et des méthodes dérivées.}

L'un des aspects les plus intéressants de cette catégorie de méthodes est leur généralité : elles permettent de traiter tous les cas qui so présentent en hydraulique (jusqu'à présent). Ainsi, on peut étudier les installations contenant des éléments siège de coups de bélier d'onde, ou d'une facon générale des éléments dont les équations sont aux dérivées partielles.

Les méthodes d'Hunwrz-Routu ne permet. tent pas de tenir comple de ces éléments. Ia méthode de Lronhard généralisio [47] ne permet pas non plus (jusqu'à présent) de tenir compte de ces systemes, sauf s'ils sont du type " onde sans perte».

Or, on reneontre fréquemment en hydraulique des systèmes régis par des ćpuations aux dérivées partielles. Ce sont :

a) Les conduites « longues » ${ }^{1}$, sieges de coups de bélier.

Dans le cas des installations hydro-électriques, elles peuvent ètre pratiquement considérées comme appartenant au type "sans pertes » (de charge). Elles sont, par contre, tres souvent à fortes pertes de charge dans les cas d'installations d'irrigation ou d'adduction d'eau.

\section{b) Les canaux.} tion.

Les canaux sont le siege d'ondes de transla-

Les bassins «à niveau réglé » des installations d'irrigation et d'adduction d'eau doivent souvent être considérées comme des canaux courts, dans lesquels il peut y avoir des ondes. Il en est de même des modeles réduits réglés en niveau d'ean (voir figure 1$)^{2}$.

\section{c) Les liaisons ílastiques.}

Souvent, les tringleries, câbles, etc, ne peuvent pas être considérés comme infiniment rigides et sont suffisamment longs pour pouvoir donner lieu à des phénomènes d'ondes. Parfois même

1. On sait que la longueur n'est pas le seul paramètre qui intervient.

2. La vitesse des ondes dans les canaux est beaucoup plus petite que celle dans les conduites, e'est pour cela que les canaux, meme conpls, sont lo siege dondes.
Lout un ensemble d'appareils mécaniques peut ètre secoué par des vibrations élastiques sans que l'on puisse localiser une pièe plus élastique que les autres.

Do plus, la méthode de Nrovist, convenablement généralisée, nous permetra d'étudier les systemes non linéaires.

On peut classer l'ensemble de ces systimes de la façon suivante :

a) Systèmes techniquement linéaires dans cerlaines limites : résistance dectrique métallique ou au carbone; capacité électrique; dash-pot à huile; certains ressorts, etc...

b) Systèmes techniquement linéaires pour les oscillations infiniment petites, mais non linéaires pour les oscillations finies (même petites) : selfinduction contenant du fer; conduite avec vanne; pertes de charge en régime turbulent, etc.

c) Systemes qu'on ne peut jamais considérer comme linéarres : régulateur par tout ou rien; appareils contenant des relais; appareils ayant un jeu; appareils ayant un frottement sec; recouvement dans les tiroirs des régulateurs, ete.

Si ces méthodes ont l'avantage de la généralité, elles permettent également do tenir compte rationnellement du comportement physicue des systèmes. Pour se rendre compte de la stabilité d'une installation, on est obligé :

- Soit d'admettre que les différents organes ou appareils se comportent d'après des équations données à priori,

- Soit de «tester » un certain nombre d'entre eux ef d'en déduire leur comportement.

Avec ces différentes données, on peut employer l'une des methodes d'investigation connues.

Les méthodes d'Hurwitz-Routh ou de LfoNHARI ne permettent pas de se rendre compte rationnellement des approximations qu'on a le droit de faire sur les équations de départ. Ainsi, loutes les adductions d'eau sont le siege de coups de bélier d'onde; quand a-t-on le droit de néghiger les ondes? Avec les méthodes autres que colles de Nyoust, on en est réduit à une estimation « au jugé ». Cette estimation est parfois suffisante, compte tenu de l'expérience de l'opérateur.

La méthode de Nyovist est bien plus puissante, car elle permet de connaitre d'une facon exacte dans quelle mesure on a le droit de faire telle ou telle approximation au départ. Ce seul tait simplifie souvent considérablement les calculs des installations de complexite moyenne. Il permet par ailleurs l'étude d'installations inaccessibles au calcul par d'autres procédés.

cette methode permet aussi, pour chaque or- 
gane, de faire correspondre une équation déterminée à un domaine déterminé d'amplitude et de fréquence; ceci permet donc de tenir compte du comportement physique exact de cet organe. On peut également employer des données expérimentales, ce qui serait impossible par ailleurs.

Exemple de domaine de validité de différentes équations (plus ou moins simplifiées) pour un mème organe.

Ce domaine de validité, tracé clans le plan amplitude-fréquence, est, en général, délini pour des excitations sinusoïdales (pour les cas non linéaires, on devrait tenir compte en plus d'autres excitations, mais on verra par la suite que les excitations sinusoïdales suffisent).

Pour une conduite fermée par une vamne, on peut employer les équations suivantes :

A) Equation linéarisée à coefficients constants :

Aa) coups de bélier en masse,

Ab) coups de bélier d'onde.

B) Equation linéarisée à paramètre variable :

Ba) masse,

$\mathrm{Bb})$ onde.

C) Equations non linéaires simplifiées :

$\mathrm{Q}=\mathrm{K} \mathrm{Y} \sqrt{\mathrm{H}}$,

$\mathrm{Q}=$ débit;

$\mathrm{H}=$ perte de charge,

$\mathrm{Y}=$ ouverture de la vanne,

Ca) masse,

Cb) onde.

D) Equations non linéarisées plus exactes :

Da) masse,

Db) onde.

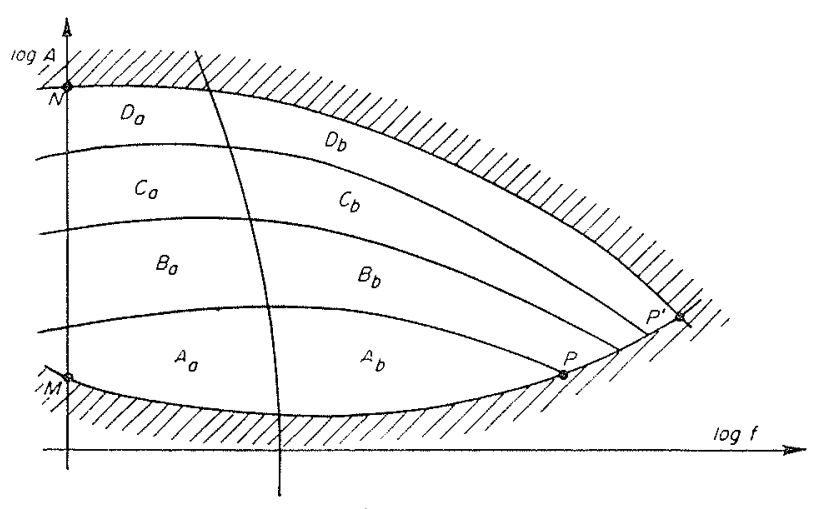

Fisi. 5.

Dans le plan amplitude-fréquence, les domaines de validité (très schématiques) sont données par la figure 5 .
Dans ces domaines, l' « erreur commise» (définie plus loin), ne dépasse pas une valeur donnée, connue à l'avance.

L'équation Aa est valable dans le domaine Aa.

L'équation $\mathrm{Ba}$ dans l'ensemble des domaines Aa et Ba, etc.

On porte sur les axes : $\log \mathrm{A}$ et $\log f$ (A : amplitude - $f$ : fréquence). Dans la région hachurée, ou bien la conduite "ne répond plus » aux excitations, ou bien il est absurde de se placer en de tels points. Les courbes ainsi tracées sont, en général, appelées courbes de Weygel; l'ensemble forme les citrons de Wrygre (à cause de la forme des courbes). La courbe MP s'appelle la courbe de « seuil », la courbe NP est celle de la «saturation».

En pratique, on assimile les « citrons» de Wexger à des rectangles (fig. 6).

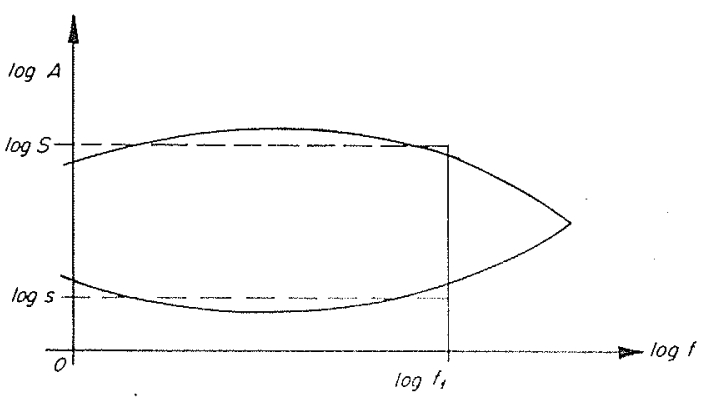

FIG. 6.

On appelle l'intervalle $o-t_{1}$ la bande de fréquence admissible ou passante; $s$, le seuil, $\mathrm{S}$, la saturation. L'axe des « $\log f$ est gradué en octaves (ou décades), l'axe des $\langle\log \mathrm{A} \gg$ en décibels par rapport à $s^{1}$. Le nombre $\log \frac{S}{s}$ est une constante essentielle d'un appareil. Pour un bon appareil de mesure électrique $\log \frac{\mathrm{S}}{s} \# 60 \mathrm{db}$ pour une trìs bonne balance de chimiste 120 à $130 \mathrm{db}^{2}$, pour un très bon moteur électrique (type OnERA) $55 \mathrm{db}$. En régulation, c'est beaucoup moins en général.

1. Une octave correspond à un rapport $\frac{f_{1}}{f_{2}}=2 ; \log$. $\log , \frac{f_{1}}{f_{2}} \# 0.3$. De inême, le nombre $D$ en décibels est défini par $\mathrm{D}=20 \log \frac{\mathrm{A}}{\mathrm{s}}$. A. A un nombre $\frac{A}{\mathrm{~s}}=2$ correspond $\mathrm{D}=6 ; \frac{\mathrm{A}}{\mathrm{s}}=5, \mathrm{D}=14 ; \frac{\mathrm{A}}{\mathrm{s}}=10, \mathrm{D}=20$, etc.

2. Cette comparaison paraît désavantageuse pour les électriciens. En réalité, la balance est un appareil différentiel comme un pont de Wheatstone. 
Dans le domaine plus limité des systèmes linéaires, la méthode de NyovisT permet de trouver assez facilement la fréquence propre la moins amortie et la valeur de son amortissement. Elle permet aussi d'indiquer s'il existe une deuxiême période propre dont l'amortissement ne serait pas beaucoup plus grand que celui de la première. En général, les systèmes de régulation ont uno période propre dont l'amortissement est nettement plus faible que celui des autres. Une excitation unique produit alors un mouvement tendant tres rapidement vers :

$$
A e^{\lambda t} \cos (\omega t+\varphi)
$$

où $\lambda$ est l'amortissement le plus faible et w la période correspondante.

On connait done le degré de stabilité. Les méthodes Hurwitz-Routh classiques ne permettent pas de connaitre l'amortissement. Leur's généralisations permettent de fixer une limite inférieure, mais les calculs sont en général beaucoup plus compliqués que l'application de la méthode d'Hurwrty ordinaire.

Pour les systèmes non linéaires, la déterninifion de l'amortissement reste toujours délicate, mais eeci est un fait inhérent à la nature de ces systèmes, comme on le verra plus loin. Néanmoins, la méthode de Nrouist est à même de donner des indications très utiles.

La méthode de Nrourst permet egalement de se faire facilement une idée génćrale de la précision du réglage et des améliorations susceptibles d'être apportées aux installations moyennement oụ très compliquées.

Il faut distinguer ici quatre cas :

a) Les systimes linéaires à « minimum de phase » (voir définition plus loin).

On rencontre très souvent ces systèmes quand on « linéarise » (voir plus loin) les problèmes de régulation. Ce n'est jamais le cas des turbines réglées en fréquence-puissance.

Pour ces systemes, il existe une methode d'avant-projet extrêmement simple.

b) Les systèmes linéaires non à minimum de phase, mais contenant des déphaseurs pur's simples.

Cest le cas de beaucoup de systèmes linéarisés ren particulier en ec qui concerne la régulation en fréquence-puissance des turbines).

\section{c) Les systèmes linéaires non à minimum de phase et a dephasenr "compliqué.}

C'est Io cas des installations a coup de bélier londe par exemple.
Il faut alors avoir recours à la méthode de Nyourst originale.

\section{d) Les systèmes non linéaires.}

En réalité, c'est le cas de tous les systèmes, c'est aussi le cas de beaucoup d'entre eux, même en première approximation.

Pour les cas b) et c), la méthode de M, Nasse est souvent intéressante [53].

La méthode de Nyourst a également permis de mettre en lumière deux sortes de stabilité. On sait que certaines installations parfaitement stables sont néanmoins à déconseiller en pratique. En effet, le vieillissement des appareils ou les petits accidents (par exemple bouchage d'un tuyau d'huile, d'un régulateur) entraine souvent une instabilité due à une diminution de l'amplification des divers organes. Grâce au diagramme de NYQurst, on peut distinguer dans ce "ass la stabilité dite sabsoluc » et la stabilité "théorique ». Ces termes sont consacrés par l'usage [34] [52]. Les autres méthodes ne permettent pas de définir ces notions.

Enfin, un autre avantage de la méthode de NyQurst est sa simplicité : elle permet de décomposer les installations automatiques en éléments simples. A chaque élément est attachée une courbe. En général, ce sont les mèmes éléments combinés différemment qui servent toujours. Le nombre de ces éléments est relativement petit en hydraulique. Il suffit chaque fois de combiner les éléments qui constituent le système antomatique pour connâ̂tre la stabilité et la précision. On peut done dresser un «catalogue » d'ćléments contenant les courbes caractéristiques de chaque Clément (courbe de transfert, de Wryger, ete.).

La méthode de Nyourst ne nécessite alors plus (que des calculs simples (rigles de trois) et des graphicques. Les problèmes de stahilité peurent alor's être facilement résolus par un bureau d'études. Les autres méthodes nécessitent à veu près toujours l'intervention de mathématiciens (ce qui n'est pas toujours sans inconvénients pour le (echnicien).

La méthode graphique est, d'ailleurs, en général, infiniment plus rapide, surtout cquand il s'agit de systèmes à minimum de phase on à déphaseur simple (systèmes dont il était déjà question plus haut).

\section{II-4. - Cas où les méthodes de Hurwitz-Routh sont intéressantes.}

Comme déjà signalé, il y a des cas où les méthodes d'HuRTitz-Rotrm, ou mème la résolution 
directe d'une équation caractéristique, sont intsressantes.

Il faut, pour cela :

a) Que le système soit linéaire;

b) Qu'il ne contienne pas d'organe siege d'ondes; c) Que le degré de son équation caractéristique ne dépasse pas 4 ;

(d) Que les perturbations soient inconnues ou du genre *échelon unité ».

Ces cas se rencontrent en hydraulique [1] [2] [3] [4], ete.

\section{III. - DESCRIPTION DES INSTALLATIONS DE REGULATION ET DÉFINITION DES TERMES EMPLOYÉS}

Les méthodes dont il est question ici (NYouist, BoDE, etc.) sont basées sur une conception nouvelle des régulateurs. Pour les comprendre et les appliquer, il faut d'abord connaître la description des installations de régulation qui est à leur base.

Les autres méthodes connues et emplovées jusqu'à présent ne tenaient pas compte des particularités très grandes des installations de régulation par rapport à ce qu'on appellera les « systèmes physiques » (définis plus bas). Ces méthodes s'appliquaient à des systèmes bien plus généraux que les installations de régulation. C'est d'ailleurs pour cela qu'elles sont d'un maniement hien plus difficile et ne présentent pas les avantages cités dans le parag. II-3.

Après la description des installations (pui nous intéressent, le point le plus important est l'analyse harmonique, c'est-à-dire l'étude des systèmes soumis à des excitations sinusoïdales. C'est pratiquement une extension des calculs avec nombres complexes dont se servent si souvent les électriciens (impédances complexes du genre : $\left(\mathrm{R}+j \omega \mathrm{L}+\frac{1}{j \omega \mathrm{C}}\right)$

La détermination de l'amortissement et de la précision met en jeu un mode de calcul analogue au calcul symbolique; le calcul symbolique n'est évidemment pas nécessaire pour la compréhension de la majeure partic de ce qui suit.

\section{III-1. - Description \\ de deux installations industrielles simples.}

$$
\text { (Cf. fig. 7) }
$$

Ces installations sont évidemment des installations industrielles, telles qu'elles se construisent. Ce sont des « stations brise-charge » pour l'adduction d'eau des grandes villes. Rappelons, en quelques lignes, leur but et leur fonctionnement.

Pour alimenter les grandes villes en eau potable, il faut parfois chercher cette eau très loin. Admettons que cette eau soit captée dans une grande retenue, dont le niveau est nettement supérieur à l'altilude de la ville. Ceci est, par exemple, le cals pour la ville de Tunis. Pour pouvoir distribuer l'eau à une pression convenable, on intercale sur la conduite d'amenée une station

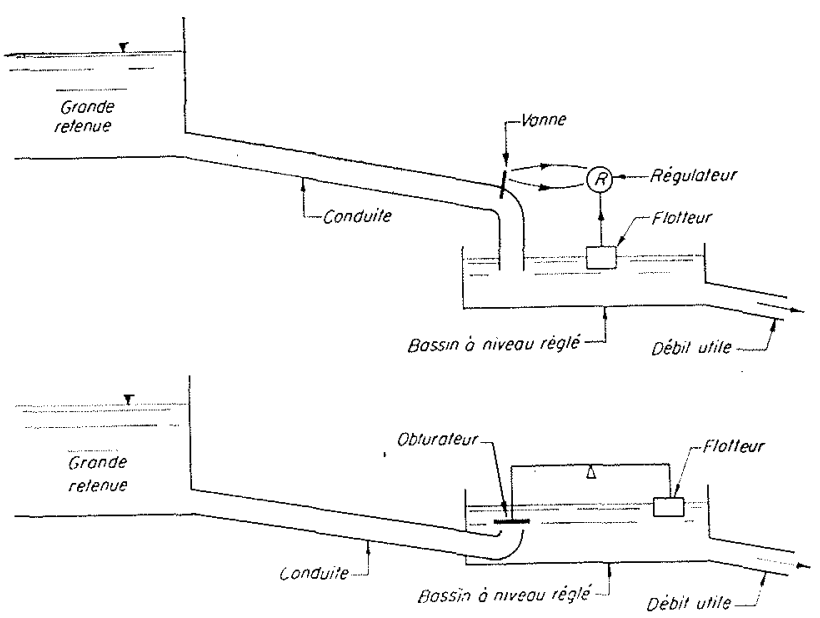

Fig. 7.

«brise-charge ». Elle doit permettre de fournir en permanence le débit demandé avec la charge imposée par un «bassin a niveau réglé 》 (roir figure 7). Le réglage de ce niveau se fait par un système de régulation, qui maintient le niveau pratiquement constant, quelles que soient les variations du débit demandé à l'aval.

Comment marchent ces stations brise-charge? :

Première station. - Le flotteur détecte le niveau du bassin aval et le transmet au régulateur. Le régulateur manouvre la vanne en conséquence.

Si le niveau est trop haut, la vanne se ferme et inver'sement.

Deuxieme station. - Le flotteur agit directement sur un obturateur (de la mème façon que plus haut).

On voit que les installations sont composées d'appareils agissant les uns sur les autres. On peut représenter ces installations d'une facon 
encore plus schématique : chaque rond symbolise un appareil, les traits les joignant indiquent les actions possibles entre les appareils.

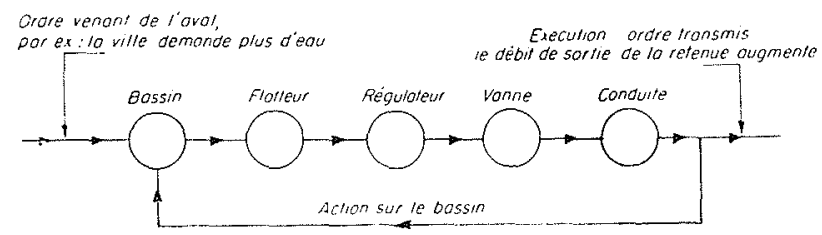

liti. 8.

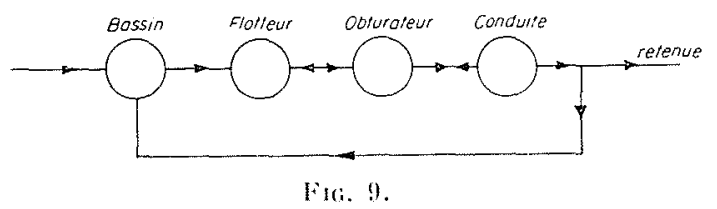

Il est trè important de bien voir qu'efrectivement chaque trait indique une action of qu'aucume autre action n'est possible.

Nous appellerons liaisons les actions possibles entre les appareils et symbolisées par les traits des figures 8 et 9. Nous allons étudier ces liaisons. Cette étude est absolument fondamenlale pour la suite.

Remarquons d'abord qu'il y a deux sortes de liaisons entièrement différentes :

a) Les liaisons diles "sans reaction 》 ou cncore « a sens unique $» ;$ nous les schématiserons par un trait et une seule fleche (fig. 10).

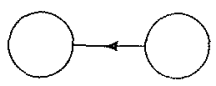

Fig. 10

Elles permettent l'action d'un appareil sur un autre, mais non inversement.

Exemple : Considérons les deux appareils flotteur et régulateur; si le flotteur bouge, il fait obligatoirement marcher le moteur du régulateur. Par contre, la marche du moteur n'a aucune actioin directe, c'est-à-dire sans l'intervention d'un autre organe, sur la position du flotteur. (Il y a une action indirecte à travers l'ensemble du système vanne, conduite, bassin, mais pas par le trait reliant directement le flotteur au régulateur.)

C'est pourquoi le trait joignant le flotteur au régulateur n'a qu'une flèche qui indique le sens de l'action possible. De même, il y a d'autres liaisons sans réaction qui sont également indiquées par une seule flèche. b) Les liaisons à réaction. Elles permetlent l'action d'un appareil sur un autre et inversement. Nous les schématiserons par un trait ef deux fleches en sens inverse (fig. 11).

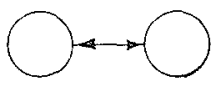

Fic. 11.

Lxemple: Considérons le deuxième schéma. Ice flolleur peut déplacer l'obturateur, mais la surpression dans la conduite soulde l'obturateur at agit sur le flotteur (fig. 12).

Pour l'ensemble des "systèmes physiques » (voir définition plus loin), les liaisons avec réacfion sont, de loin, les plus nombreuses ${ }^{1}$. Par con-

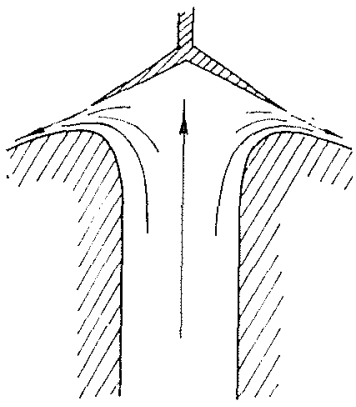

FIG. 12.

tre, dans les installations de régulation, il y a toujours au moins trois liaisons sans réaction dont on verra la disposition plus loin. Ceci est justement une des particularités des installations à régulation automatique. Expliquons pourquoi il $y$ a obligatoirement des liaisons sans réaction dans les installations de régulation. Pour donner un ordre correctement, il faut que l'organe auquel on donne cet ordre ne se « raidisse pas », c'est-à-dire ne réagisse pas. Il faut que cet organe soit «docile » et transmette cet ordre sans la moindre réaction sur l'appareil qui le lui donne.

Donnons une explication plus physique : on veut faire varier arbitrairement le débit dans un canal en agissant à l'amont de celui-ci. Si l'écoulement $y$ est quelque part torrentiel, c'est-à-dire s'il ne permet pas de réaction de l'aval sur l'amont, ceci est facile. Par contre, si l'écoulement est partout fluvial et permet, par conséquent, la réaction de l'aval sur l'amont, ceci n'est plus possible: le débit dépend outre de l'amont, aussi de la bonne ou mauvaise volonté de l'aval.

Après avoir décrit les liaisons entre organes,

1. En réalité, il n'existe en toute rigueur pas de liaison sans aucune réaction, ce n'est rqu'une idealisalion. (Voir plus loin dans le \& III-4.) 
étudions ces derniers. On classe les divers organes ou appareils en deux catégories :

a) Ceux qui ont deux liaisons avec l'extérieur :

Exemple : flotteur, régulateur, vanne, obturateur...

b) Ceux qui ont trois liaisons avec l'extérieur :

Exemple : bassin...

On constate que :

1) Dans toutes les installations de régulation hydraulique, il n'existe que ces deux types d'organes;

2) Les organes à trois liaisons ont toujours une position spéciale que nous préciserons par la suite.

Définition : On appelle élément, un organe ou un ensemble d'organes couplés ensemble et ayant chacun deux liaisons et tel que cet organe ou ensemble débute ef finil par une liaison sans réaction.

Des organes el des éléments, seuls ces derniers ont une individualité suffisante pour qu'on puisse lacilement étudier l'ensemble de l'installation en les prenant seuls en considération.

Comme on l'a vu, les installations de régulation contiennent toujours des liaisons sans réaction; il est done toujours possible de les décomposer en éléments.

Les deux inslallations (fig. 8 et 9 ) contiennent les éléments suivants :

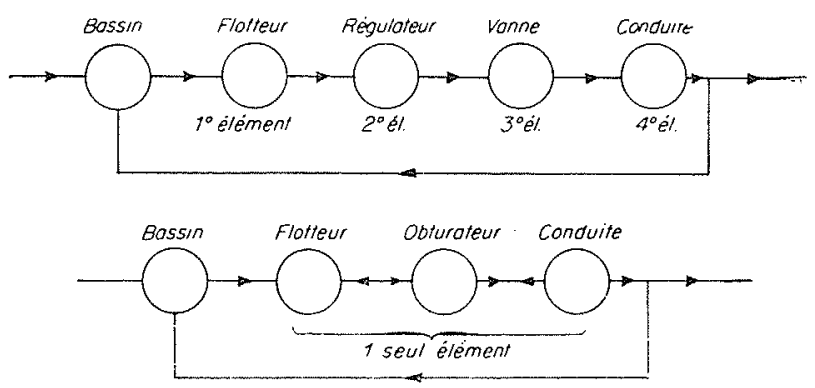

Fig. 13 .

Le bassin ayant trois liaisons, ne peut pas fairc partie d'un ćlément ou être un élément.

Etudions plus en détail ce dernier organe. Nous voyons (fig. 13) que les installations schétamisées contiennent chacune des éléments et un bassin. D'une facon générale, toutes les installations de régulation contiennent des éléments et des organes à trois liaisons avec l'extérieur. Ces derniers organes ont toujours le même rôle; ils comparent deux grandeur's et font agir en conséquence le régulateur.
Exemple : L’équation du bassin (fig. 13) est :

$$
\mathrm{s} \frac{d \mathrm{~N}}{d t}=q_{s}-q_{e}
$$

$q_{s}:$ débit sortant;

$q_{0}:$ débit entrant;

$\mathrm{S}$ : surface du bassin;

$\mathrm{N}$ : niveau (compté positivement vers le bas).

Si $q_{s}=q_{c}$, le régulateur est à l'arrêt.

Selon que $q_{s} \gtrless q_{s}$, le régulateur ouvre on ferme la vanne.

Pour l'ensemble de ces raisons, on appelle les organes analogues au bassin de la figure 13 des comparateurs ou discriminatenrs.

Pour des commodités de raisonnements, on définit le comparateur idéal : le comparateur compare le débit entranl $q$. el le débit sortant $q$. en agissant sur le flotteur par la grandeur $q=q_{\mathrm{s}}-q_{\mathrm{c}}$.

En réalité, les comparateurs établissend en général une relation plus compliquée. Ainsi, un bassin donne en plus la relation :

$$
s \frac{d \mathrm{~N}}{d t}=q \text {. }
$$

On décompose alors le bassin en un comparateur idéal et un élément fictif qui établit la relation voulue. Ceci sera expliqué plus en détail au $\$$ III-6.

Les deux installations simples de la figure 7 se schématisent finalement comme suil :

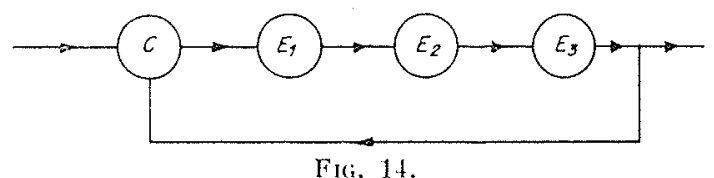

C est le comparateur,

$\mathrm{E}_{1}, \mathrm{E}_{2}$, etc., sont les éléments.

L'ensemble a la forme d'une boucle fermée. On l'appelle la boucle de régulation. Les installations relativement simples (et de loin les plus nombreuses en hydraulique) sont eonstituces par une seule boucle ${ }^{1}$.

Cette boucle contient toujours un comparateur et un certain nombre d'éléments, comme le montre la figure 14. Elle ne contient jamais autre chose.

1. En réalite, l'asservissement est aussi une "petite boucle " mais elle n'offre jamais de difficultés spéciales. 


\section{III-2. - Les différents régulateurs employés.}

Outre les régulateur's à une boucle, il existe beaucoup d'autres sortes de régulateurs.

Régulateurs à plusieurs boucles (fig. 15) :

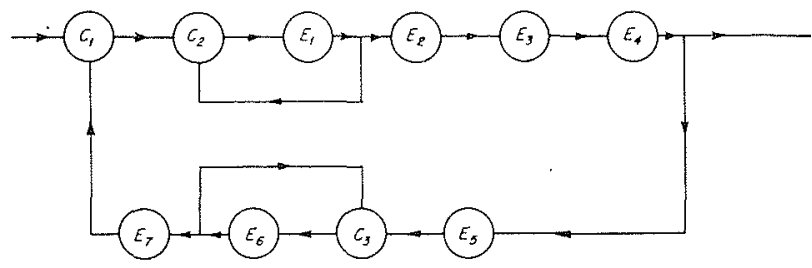

F1G. 15.

$\mathrm{C}_{1}, \mathrm{C}_{2}, \mathrm{C}_{3}$, sont des comparateurs.

$\mathrm{E}_{1}, \mathrm{E}_{2}, \mathrm{E}_{3}$, sont des éléments.

On distingue la boucle principale et les boucles secondaires, tertiaires, etc... Ces régulateurs s'appellent régulateurs à boucles.

On constate que :

1. Toutes les installations de régulation employées en hydraulique (et probablement d'une façon générale en technique) sont des régulateurs à boucles;

2. Une boucle de régulation ne contient que des éléments, éventuellement des boucles d'ordre inféricur, el un comparateur.

Les méthodes exposées ici s'appliquent essentiellement aux régulateurs à boucles.

On peut concevoir des régulateurs bien plus compliqués que les régulateurs à boucles. La figure 16 en donne un exemple.

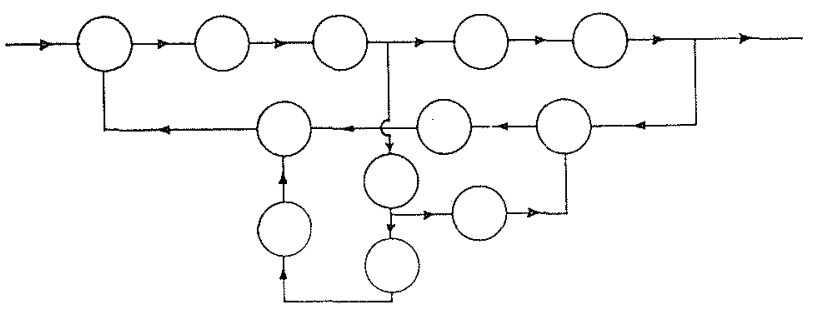

FIG. 16.

Ces régulateurs sont à connexion maillée. Ils ne rentrent pas dans le cadre des méthodes employées ici. A notre connaissance, de tels systèmes n'ont pratiquement jamais été employés en hydraulique. Néanmoins,. si on veut les étudier, on peut voir [37].

Régulateurs réglant plusieurs paramètres. Il y en a deux sortes : (t) Ceux qui sont composés de la juxtaposition de plusieurs régulateurs à un paramètre. IIs ne posent pas de problemes vraiment nouveaux.

Exemple : Régulation de fréquence et de tension des réseaux de distribution électrique;

b) Ceux qui ne sont pas de simples juxtapositions.

Leur étude sortirait du cadre de cet article; nous ne faisons que les mentionner ici [54].

\section{III-3. - Décomposition des éléments des boucles de régulation.}

Rappelons qu'un élément est un ensemble d'organes couplés à deux liaisons et qui débute et linit par une liaison sans réaction (fig. 17).

Nous allons étudier d'une façon plus précise quels sont les organes susceptibles d'entrer dans la composition d'un élément; et aussi quelles

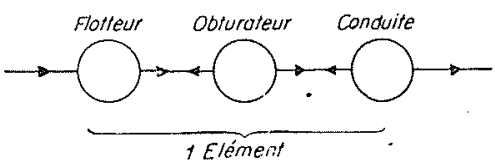

Fig. 17.

sont les proprićtés de lels organes. On en déduiral ensuite les propriétés des éléments.

Les organes et appareils intervenant dans les installations de régulation font partie de la grande famille des «systimes physiques». C'est pour cela que nous allons commencer à décrire et classer les « systèmes physiques » en général.

Employé par plusieurs auteurs [26] [65], ce mot de «systèmes physiques » n'a pas, à notre connaissance, été nettement défini; nous essayerons, ici, de préciser sa signification ${ }^{2}$.

Un « système physique » est un ensemble de corps occupant une région de l'espace, limitée par une frontière bien définie; e'est là une caractéristique géométrique, commune d'ailleurs à un grand nombre de systèmes. L'intérieur de cet ensemble est le siège de phénomènes physiques. Mais, en outre, de par sa structure interne, un système physique ne reste pas indifférent à l'espace environnant; il établit sur ses frontières ou sur une partie de ses frontières des relations entre des grandeur's physiques (et c'est là sa caractéristique essentiellement physique); on dit

1. Nous n'entrerons pas dans les détails, étant donné que ces questions dépassent les problème qui nous préoccupent. 
alors qu'il y a cntre lui et l'espace extérieur: des liaisons. Ces liaisons sont exprimées mathématiquement par les relations en question.

Ainsi, cette notion de "système physique * déborde largement le cadre de la régulation. Des exemples permettront de mieux saisir la définition nécessairement générale donnée ci-dessus.

Considérons une conduite d'eau; elle est formée de la conduite proprement dite et du fluide (l'eau) (qui s'icoule par cette conduite. Elle constitue un «systime physique». Ses frontières sont, d'une part, les parois, d'autre part, les sections d'entrée et de sortie. Les grandeurs physiques intéressantes sont le débit et la pression; les débits et pressions sur les frontières sont liés par une relation (Bervouili, par exemple).

De mème, un port de mer, une self-induction électrique constituent des «systimes physiques».

On classe généralement les systèmes physi. ques d'après le nombre de dimensions spatiales, ou, d'une facon plus exacte, d'apres le nombre minimum de coordonnces intervenant dans les équations régissant les phénomènes physicques considérés.

Ainsi, dans les équations de propagation de la houle à l'intérieux d'un port, trois coordonnées interviennent : c'est un « système physique $\gg \dot{a}$ trois dimensions. Les équations de l'écoulement de l'eau dans une conduite font intervenir une seule coordonnée (l'abscisse curviligne de la ligne moyenne de la conduite : une conduite sera classée « système physique » à une dimension 1$)$.

Une self électrique ou un moteur au contraire ne font intervenir aucune coordonnée spatiale; on les classe comme « systèmes physiques à constantes localisées ». Pourquoi cette dénomination bizarre? Sur une ligne électrique, la selfinduction est répartie le long de la ligne; au contraire, dans un appareil électronique, la self est localisée au point où on la place. D'où le nom de « constante Jocalisée ».

Ainsi apparaissent quatre classes de systèmes physiques :

1. En toute rigueur, il y a evidemment toujours trois dimensions. Ial discussion des cas où on peut ne tenir compte que de une ou deux dimensions deborde le cadre de eet article. Rappelons néanmoins que non seulement les caractéristiques du systeme, mais aussi celles de lexcitation interviennent. Pour chaque systeme, on peul lracel dans un plan amplitude-frétuence des domaines de validite des differentes approximations (ct. courbes de Weygel). Une caracteristique essentielle pour pouroir négliger une dimension est le rapport du temps de propagation d'une onde suivant eette dimension au temps fondamental de l'excitation; néanmoins, pour certaines excitations, ee rapport ne joue pas exemple : thérie des prismes infinis non sollicites parallelement a leurs gencmallices).
a) A trois dimensions,
b) A deux dimensions,
c) A une dimension,
d) A constante localisée.

Si l'on compare le nombre de dimensions d'un système avec le nombre de dimensions de sa frontière, on trouve les résultats indiqués dans le tableau suivant :

\begin{tabular}{|c||c|}
\hline \multicolumn{1}{|c||}{ Srstemes } & Frovitires \\
\hline 3 dimensions & 1 dimension \\
2 & 2 dimensions \\
Constante local. & ponctuelles \\
\hline
\end{tabular}

Les systèmes des deux dernières classes ont les mêmes frontières, e'est pourquoi ils présentent un grand nombre de proprićtés communes (par exemple, ils ont une impédance).

Frontrikres. - Il convient maintenant de revenir sur la notion de frontières.

Prenons l'exemple d'un port, nous remarquons que la frontière (digues, fond, surface libre, passe d'entrée) ne constitue pas un tout uniforme : les digues et le fond isolent le fluide de l'espace extérieur, de même à travers la surface libre il n'y a en général pas d'action *; la passe d'entrée, au contraire, permet la communication avec l'extírieur; sur les digues, il n'y a pas, a proprement varler, relation entre la hauteur d'eau et le débit; dans la passe d'entréc, au contraire, ces deux grandeurs sont liees par une loi ef quand une de ces grandeurs variera, l'autre variera conformément à cette loi. Aussi, nous sommes amenés à distinguer différentes sortes de frontières : les digues, le fond et la surface libre sont des frontieres hermétiques ou fixes, la passe d'entrée une frontiere mobile. Autrement dit, les frontières des systèmes à deux ou trois dimensions peuvent être partiellement bloquées (digues, fond), les frontières restantes étant libres. Ces frontieres libres peuvent se réduire à des points. Ces systèmes ont - - vus de l'extérieur -... des propriétés analogues à celles des systèmes à constantes localisées ou à une dimension. On appelle l'ensemble de ces systèmes les systimes à liai-

2. En faisant abstraction du rent. 
sons ponctuelles avec l'extérieur, systèmes que nous examinerons plus attentivement à l'aide d'exemples empruntés au sujet mème de notre article, c'est-à-dire aux régulateurs. Les installations de régulation ne contiennent que des "systèmes physiques à liaisons ponctuelles avec l'extérieur $\gg$. Signalons cependant une remarque relative aux liaisons analogues à celle faite au sujet des dimensions : e'est uniquement l'équalion qui fait apparaittre la liaison ponctuelle, mais en réalité cette liaison a toujours plusieurs dimensions.

Exemples de systemes a liaisons ponctuelles.

Nous énumérerons un certain nombre de ces systemes qui se rencontrent en régulation (voir en particulier fig. 7).

\section{a) Systimes a constante localistá.}

Régulateur : Lorsque la tíge du flotteur se déplace, ce déplacement agit sur le régulateur; cette action est dite "action d'entrée » ou, dans un langage plus concis encore, "entrée ».

De mème le régulateur va agir à son tour sur la poulie du moteur de la vanne; on désigne cette action sous le nom de « sortie ».

Floteur : Avec la mème terminologie « entrée et «sortie», on aura pour le flotteur :

- Pour l'entrée : déplacement du centre de poussée hydraulique.

- Pour la sortie : tige du flotteur.

\section{Vanne :}

Pour l'entrée : position de la vanne;

Pour la sortie : relation perte de charge/débit.

\section{b) SYstimes a d DE DIENSION.}

Pour l'exemple de la conduite reoup de bélier l'onde), on a pour :

- Entré : position de la vanne;

- Sortie : débit de sortie.

\section{c) SYSTHMES A DEUX DIMENSIONS, MAIS A LIASONS PONCTUELLES.}

Nous avons vu que les systimes physiques à constantes localisées et ceux à une dimension ont toujours des liaisons poncluelles. Les systimes à deux ou trois dimensions peuvent ígalement avoir des liaisons ponctuelles avee l'extérieur. C'est d'ailleurs uniquement de cette façon qu'ils interviennent en régulation.

Exemple : Un grand bassin rectangulaire soumis à un mouvement sinusoïdal.
Les équations font bien intervenir deux coordonnces spatiales dans le plan, mais les entrées et sorties peuvent être considérées comme ponetuelles.

Il se peut que plusicurs systimes physiques simples aient des liaisons à plusieurs dimensions, mais on peut toujours trouver un systeme plus grand les englobant et ayant des liaisons poncluelles.

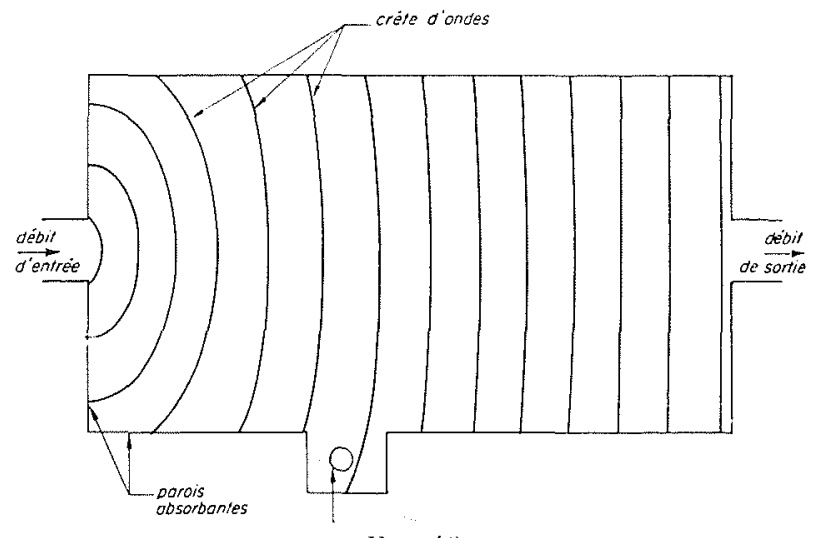

Fiti. 18.

Pour chasser correctement les systemes à liaisons ponctuelles, il faut d'abord élucider un point délicat à propos des grandeurs intervenant dans les systemes physiques en géneral.

On a vu qu'un système physique établissait (par sa structure interne) certaines relations entre les grandeurs mesurées sur ses limites.

Exemple : Un réseau d'adducion d'eau établit des relations entre les pressions et les débits d'alimentation et de distribution. Ine poutre établit une relation entre les charges qu'elles portent el ses déplacements, ete. On constate que, dans tous les systènes physiques, il existe deux sortes de grandeur ${ }^{1}$ :

1. Les grandeur's diles de «tension»;

2. Les grandeurs dites de « déliit ».

\begin{tabular}{|c|c|c|}
\hline FXEMPLE & TENSION & DÉBIT \\
\hline Electricité & tension. & courant \\
\hline Mécanique & foree. & ritesse \\
\hline & $\begin{array}{l}\text { pression, } \\
\text { charge. }\end{array}$ & débit \\
\hline Thermodynamique & temperature. & entropie \\
\hline
\end{tabular}

1. Nous n'avons nullement l'intention de discuter iei la valeur des analogies physiques voir a ce sujet, par exemple: $[43],[15],[28]$, 55]). Nous voulons simplement nous en servir. 
Ces grandeurs ne sont pas arbitraires : l'une est du type «covariant ", l'autre du type « contravariant $»$; leur produit est une puissance.

La tension est toujours mesurée par rapport à une tension de référence; cette référence est, en électricité : "la masse »; en mécanique : "le trièdre de référence »; en hydraulique : "le plan de référence des charges »; en thermodynamique : «le zéro de température».

Ce «zéro » de référence lie le systime à l'extérieur (mathématiquement et surtout aussi physiquement). Il faut absolument tenir compte de cette liaison. Parfois elle existe matériellement, parfois elle est invisible; c'est ce qui rend difficile le décompte du nombre des liaisons ponctuelles. Ceci rend aussi difficiles les « analogies » physiques entre systèmes électriques et mécanique par exemple. En électricité, la liaison "zéro de tension » est un fil, en mécanique, c'est un « triedre invisible».

Nous compterons systématiquement le «zéro de tension » comme une liaison, mème s'il n'est pas visible.

Il ne peut pas alors exister de système à une seule liaison ponctuelle. Les systèmes à deux liaisons ponctuelles s'appellent dipôles.

Exemple: (A et $\mathrm{B}$ sont des liaisons).

Self (fig. $19 a$ );

Pendule (fig. $19 \mathrm{~b}$ );

Chemince d'équilibre (fig. $19 \mathrm{c}$ ).

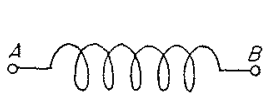

Fici. $19 a$.

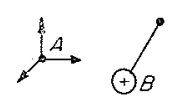

Fic. $19 \mathrm{~b}$.

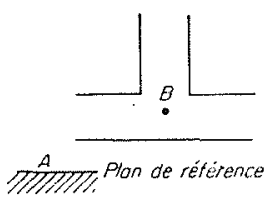

Fig. 19 c.
On définit de même les tripôles, quadripôles, cte. En réalité, on ne parle jamais de tripôles; on les classe avec les quadripôles. Les tripôles n'ont pas de propriétés plus simples que les quadripôles.

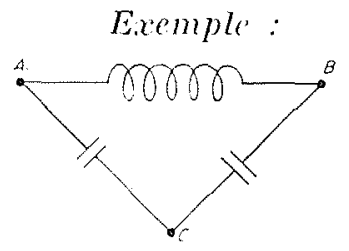

FIG. 20.

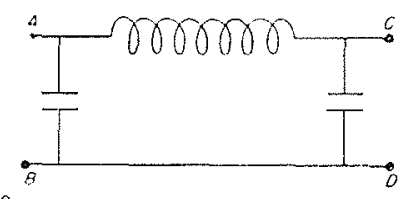

Ce que nous avons appelé liaison dans les $n$ "III-1 et suivants, ne constituaient pas, en réalité, les uniques liaisons des organes envisagés. Ces organes sont, en réalité, tous des quadripôles (ou des tripòles) (voir fig. 21); on ne parlera, par lar suite, plus que de quadripòles.

Les quadripôles ont un débit d'entrée et un débit de sortie $I_{n}$ el $I_{s}$ : de même deux tensions $\mathrm{U}_{e}$ et $\mathrm{U}_{s}{ }^{1}$ :

Exemple : Condurte (fig. 21 a) :

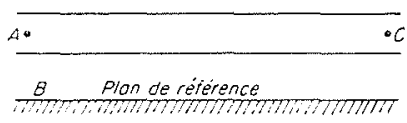

Frg. $21 a$.

rension : cntrie : charge $A$, sortie : charge C.

Débits : entrcée : débit A, sortie : débit $\mathrm{C}$.

Exemple : Motedr fítectrigue (fig. $21 \mathrm{~b}$ ).

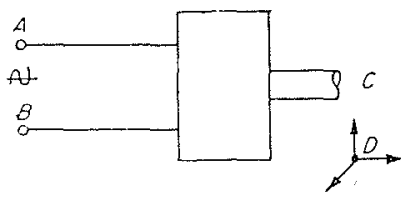

Fic. $21 \mathrm{~b}$.

Tension : entrée : électrique, sortie : couple mécanique.

Débits : 'ntrée: courant álectrique. sortie : vitesse de rotation.

Exemple: Orturateun (fig. $21 \mathrm{c}$ ).

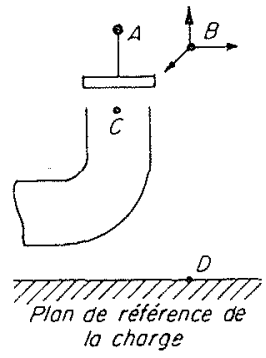

Fiti, $21 c$

Tension : entrée : force, sortie : charge.

Dábits : entrée: vitesse de déplacement, sortie : débit d'eau.

On constate que les éléments de régulation ne contiennent que des quadripôles.

1. Une discussion serait à laire, ì ce sujet, comme on sait; nous la passons ici. 
C'est pour cela que nous allons étudier plus en détail ces systèmes physiques.

\section{III-4. - Les quadripôles généralisés.}

L'état d'un quadripòle est défini par sa tension d'entrée $U_{t}$, sa tension de sortie $U_{s}$ et ses débits d'entrée el de sortie $i_{e}$ et $i_{s}$.

Le quadripôle établit (de par sa structure interne) deux relations entre ces quatre grandeurs. Ces deux relations caractérisent le quadripôle. Admetions qu'elles soient :

$$
\begin{aligned}
& \mathrm{U}_{b}=f_{1}\left(i_{b} ; i_{s}\right) \\
& \mathrm{U}_{s}=f_{2}\left(i_{b} ; i_{s}\right)
\end{aligned}
$$

Nous allons, maintenant, classer les quadripôles d'après la nature de leurs relations fondamentales.

Définissons la nature d'une relation en général.

On dit qu'une relation :

$$
\Phi\left(x ; y ; z ; \frac{d x}{d t} ; \frac{d y}{d t} ; \frac{d z}{d t} ; \frac{d^{2} x}{d t^{2}} \ldots\right)=0
$$

est linéaire si elle se met sous la forme :

$$
\begin{aligned}
& a_{11} x+a_{12} y+a_{13} z+\ldots \\
+ & a_{21} \frac{d x}{d t}+a_{22} \frac{d y}{d t}+\ldots \\
+ & a_{31} \frac{d \ddot{*} \cdot}{d l}+\ldots
\end{aligned}
$$

les $a_{i j}$ étant des constantes ou des fonctions de $t$ seul ${ }^{2}$.

On dit qu'une relation $\Phi(x, y)=0$ est linéarisable pour $x=x_{0}, y=y_{0}$ si la courbe représentant $\Phi(x, y)=0$ dans le plan $(x, y)$ admet une tangente et une seule au point :

$$
x=x_{0} ; \quad y=y_{0}
$$

De même, pour une relation $\Phi(x, y, z)=0$, la surface représentative doit admettre un plan tangent, et un seul.

De même pour :

$$
\Phi\left(x ; y ; z ; \frac{d x}{d t} ; \frac{d y}{d t} \cdots\right)=0
$$

1. On ne peut pas toujours les mettre sous cette forme, mais les conclusions ulterieures restent.

2. En réalite, on admet en général, sans le dire. que les $a_{i j}$ sont des constantes. Le cas où les $a_{i j}$ dépendent de $t$ sera traité avec les cas non linéaires; en effet, les theories classiques des systemes dits « linéaires» ne s'appliquent pas (impédance symbolique, critère de $\mathrm{Ng}$ quist, etc.).
Les autres relations sont dites « non linéarisables $\gg$ au point envisagé.

Un quadripòle est linéaire si ses deux relations fondamentales sont linéaires. Il est linéarisable si les deux relations sont linéarisables ou si une est linéaire, l'autre linéarisable. Autrement il est non linéarisable.

La mème classification peut ètre faite pour tous les systèmes physiques.

On voit que cette classification est purement mathématique. Il se trouve, néanmoins, que les systèmes linéaires ont des propriétés physiques remarquables. En outre ce sont pratiquement les seuls qui se laissent aborder par le calcul. Il se trouve qu'en se qui concerne les régulateurs et cn employanl la méthode de Nroust, les systèmes non linéaires se laissent traiter presque de la même facon que les systèmes linéaires. C'est pour cela qu'on peut les ćtudier dans ce cas très particulier.

Puisque les systimes non linéaires vont ètre traités d'une facon analoğue aux systèmes linéaires, nous commencerons par étudier ces derniers.

Linéarisation des quadripóles linéarisables.

Si une relation $y=f(x)$ est linéarisable, près du point $x=x_{10}, y=y_{0}$, on peut écrire :

$y=y_{0}+f^{\prime}\left(x_{0}\right)\left(x-x_{0}\right)+\frac{1}{2 !}\left(x_{0}\right)\left(x-x_{0}\right)^{2}+\ldots$

Quand on ne s'intéresse qu'aux valeurs telles que $\left(x-x_{0}\right)<\varepsilon \quad\left(y-y_{0}\right)<\eta_{0}$, on peut, en première approximation, écrire :

$$
y=y_{0}+f^{\prime}\left(x_{0}\right)\left(x-x_{0}\right)
$$

On obtient une relation linéaire, c'est ce qu'on appelle linéariser une relation.

On peut de même linéariser un quadripôle linéarisable. Par la suite, tout ce qu'on dira au sujet des systèmes linéaires s'applique également aux systèmes linéarisés?.

Jusqu'à présent, nous avons fait surtout de la description. Nous allons maintemant entrer dans la partie plus mathématique de cet exposé. Les systemes linéaires s'étudient par l' « analyse harmonique », e'est-à-dire par l'étude de leurs oscillations sinusoïdales. Nous allons d'abord définir quelques expressions utilisces, puis nous allons montrer que la connaissance des oscillations forcées sinusoïdales suffit pour connaitre entièrement le quadripôle linéaire.

Il existe dans la littérature beaucoup de livres sur les quadripôles [8] [12] [25]; pour les systèmes en général, on peut également voir [10] et $[13]$.

Rappelons que l'analyse harmonique se confond pratiquement avec le calcul des impédances com-

1. Tant que $x-x_{y}<x$ ol $y-y_{0}<\|_{0}$ 
plexes en électricité [6] [25]. Ainsi le schéma figure 22 se traduit en régime établi, par :

$$
\text { I. } \frac{d i}{d t}+\mathrm{R} i=\mathrm{E}_{0} \cos (1) i
$$

ou par :

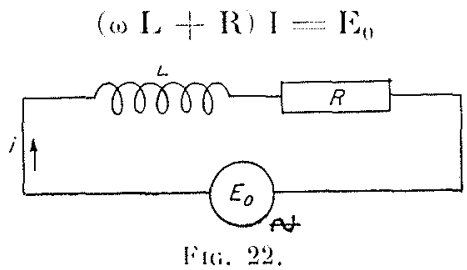

Pour étudier to quadripole, nous ferons varier par exemple $i_{e}$ sinusoïdalement. Cette variation s'appelle l'excitation ou le signal d'entrece La variation de $\mathrm{U}_{e}$ ou $\mathrm{U}_{s}$, qui en est la conséquenec, s'appelle la réponse.

Montrons qu'un quadripôle est entièrement défini par l'ensemble de ses réponses aux excitations sinusoïdales. Pour montrer ceci, nous nous servirons du principe de superposition des états d'équilibre dynamiques. Ce principe dit :

Si :

$$
U_{t 1}(t) ; U_{s 1}(t) ; i_{c t}(t) ; i s,(t)
$$

sont quatre fonctions satisfaisant anx équations (1) de ha page précédente, et si de mème:

$$
\mathrm{U}_{t: 2}(t) ; \mathrm{U}_{*: 2}(t) ; i_{r: 2}(t) ; i_{* 2}(t)
$$

satistoml aux cqualions (1),

les fonctions :

$\mathrm{C}_{1} \mathrm{U}_{61}(t)+\mathrm{C}_{2} \mathrm{U}_{022}(t) ; \mathrm{C}_{1} \mathrm{U}_{s 1}(t)+\mathrm{C}_{22} \mathrm{U}_{* 2}(t)$, ele.

satisfont également à ces équations, $\mathrm{C}_{1}, \mathrm{C}_{.}$, etc. étant des constantes.

On vérifie facilement ce principe directement.

Que veut dire : « un quadripoile est défini par... »? Cela veut dire : étant donné $i_{c}(t)$ et $i_{s}(t)$ quelconques, on peut déterminer les fonctions $\mathrm{U}_{\mathrm{c}}(\boldsymbol{t})$ ef $\mathrm{U}_{\mathrm{s}}(\boldsymbol{t})$ correspondantes. Si on connait la réponse du quadripòle à toutes les excitations sinusö̈dales, on commence à décomposer $i_{*}(t)$ et $i_{e}(t)$ en sinusoïdes démentaires ${ }^{1}$. On cherehe les réponses pour chaque sinusoide élémentaire; il suffit de recombiner les réponses pour avoir $\mathrm{U}_{\theta}(t)$ et $\mathrm{U}_{s}(t)$. Au lieu de prendre comme fonctions fondamentales les sinusoïdes, on pourrait prendre d'autres fonctions, par exemple les fonctions de Besser. Seulement, les sinusoïdes donnent les

1. Ceci est pratiquement toujours possible; si $i$ ( $l$ ) el $i$ : (t) sont périodiques, on obtient des séries de Fourrier; sinon, on obtient une intégrale de Fourrier. résultats les plus simples (à cause de leur liaison avec les équations différentielles linéaires).

ETUde des guadripòles fa réghe sinusö̈da.

Il suffit maintenant de poser :

$$
\begin{aligned}
& i_{c}=\mathrm{I}_{e} \cos \omega t \\
& \left.i_{s}=\mathrm{I}_{s} \cos (\omega) t+\vartheta\right)
\end{aligned}
$$

ct déterminer $u_{c}$ et $u_{*}$ pour définir le quadripoile.

Ici, nous arrirons au point le plus délicat de (el arlicle. Au lieu de poser :

$$
i_{c}=\mathrm{I}_{t} \cos (1) t
$$

on icrit :

$$
i_{c}=\mathrm{I}_{c} \mathrm{R}\left(e^{j \omega t}\right)
$$

R (...) veut dire : partic réelle de...

Fin cffet :

$$
e^{i \omega t}=\cos (1) t+j \sin \omega t
$$

l'oì :

$$
\mathrm{R}\left(e^{i \omega t}\right)=\cos (1) t
$$

On écrit alor's :

$$
i_{s}=\mathrm{I} \mathrm{R}\left(e^{j \omega t+\omega}\right)=\mathrm{R}\left(\mathrm{I}_{\omega} e^{j \omega t+\varphi}\right)
$$

On peut inclure $e^{j}$ dans $I_{*}$ et écrire:

$$
i_{s}=\mathrm{R}\left(\mathrm{I}_{\mathrm{s}} e^{j \times x^{t}}\right)
$$

Done :

$$
\begin{aligned}
& i_{e}=\mathrm{R}\left(\mathrm{I}_{\rho} e^{j \omega t}\right) \\
& i_{s}=\mathrm{R}\left(\mathrm{I}_{s} e^{j \omega t}\right)
\end{aligned}
$$

Que signifie le nouveau $I_{*}$ dans lequel on a inclu $e^{j_{\varphi}}$ ? $I_{s}$ est maintenant un nombre complexe. I! a gardé sa valeur absolue mais possède un alroment $\varphi$.

La valeur absolue donne toujours la valeur correcte de l'amplitude, l'argument $९$ donne le déphasage entre $i_{\theta}$ et $i_{s}$. On peut choisir une orisine de temps arbitraire telle que $i_{f}$ devienne proportionnel à cos $(\omega t+\downarrow)$. Alors $I_{r}$ et $I_{s}$ sont deux nombres complexes indicuant l'amplitude ct la phase par rapport au zéro de phase arbitraire.

On en tire :

$$
\begin{aligned}
& \frac{d i_{t}}{d t}=\mathrm{R}\left[j(1) \mathrm{I}_{\theta^{\prime}} e^{j \omega t}\right] \\
& \frac{d^{n} i_{\sigma}}{d t^{n}}=\mathrm{R}\left\{(j \omega)^{n} \mathrm{I}_{e} e^{j \omega t}\right\}
\end{aligned}
$$

de mème pour $i_{s}$. 
D'où ${ }^{1}$ :

$$
\begin{aligned}
& u_{e}=\mathrm{R}\left\{\left[\mathrm{A}_{11}(j \omega) \mathrm{I}_{e}+\mathrm{A}_{12}(j \omega) \mathrm{I}_{s}\right] e^{j \omega t}\right\} \\
& u_{s}=\mathrm{R}\left\{\left[\mathrm{A}_{21}(j \omega) \mathrm{I}_{\varepsilon}+\mathrm{A}_{22}(j \omega) \mathrm{I}_{s}\right] e^{j \omega t}\right\}
\end{aligned}
$$

Les fonctions $A_{i k}(j \omega)$ proriennent de la mise en facteur de $\mathrm{I}_{\varepsilon} e^{i \omega t}$ et $I_{s} c^{i \omega t}$.

$u_{e}$ et $u_{s}$ sont également sinusö̈daux et ont la même fréquence que $i_{c}$ et $i_{s}$. Leur amplitude est visiblement donnée par les valeurs absolues de:

$$
A_{11}(j \omega) I_{6}+A_{12}(j \omega) I_{s}
$$

et :

$$
A_{2 \mathrm{~L}}(j \omega) \mathrm{I}_{e}+A_{22}(j \omega) I_{s}
$$

puisque le facteur $e^{j \omega t}$ ne change pas la valeur absolue, mais uniquement les arguments.

De même, les phases de $u_{f}$ et $u_{s}$ sont données par ces deux expressions. En definissant $U_{t}$ et $U_{s}$, deux nombres complexes ayant pour valenr absolue l'amplitude de $u_{\theta}$ et $u_{s}$ et comme argument la phase, obtient done :

$$
\begin{aligned}
& \mathrm{U}_{c}=A_{11}(j(1)) I_{0}+A_{12}(j(1)) I_{s} \\
& U_{s}=A_{21}(j(1)) I_{c}+A_{22}(j(1)) I_{s}
\end{aligned}
$$

$\mathrm{U}_{e}$ et $\mathrm{U}_{s}$ sont alors des nombres complexes de la même facon que $I_{t}$ el $I_{s}$.

On les schematise par des vectemes dans le plan complexe (ig. 23$)$.

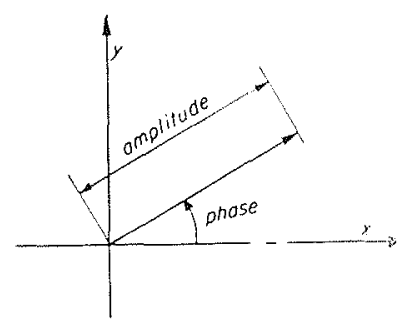

Fig. 23.

Ces quatre fonctions $A_{i k}(j \omega)$ caractérisent, comme on l'a vu, entièement le quadripòle.

On démontre que si le quadripôle est à constantes localisées, Ies $A_{i k}(j \omega)$ sont des fractions rationnelles de $j$ (1). Si le quadripôle est un système physique à une dimension, les $A_{i k}(j \omega)$ sont des fractions rationnelles de fonctions entières (comme cas particulier important, les $A_{i k}(j \omega)$ ne dépendent que de $j \omega$ et de $\left.e \sqrt{\alpha+\beta j+\gamma \omega^{2}}\right)$.

On voit que la différence entre les diverses

1. On sait qu'on ne peut pas toujours mettre les relations fondamentales sous cette forme, mais ceci est sans importance ici. classes de systénes à liaisons ponctuelles est tres petite arec colte méthode.

Nous écrilons :

$$
A_{i k}(j \omega)=a_{i k}(\omega)+j \alpha_{i k}(\omega)
$$

$a_{i k}$ el $\gamma_{i j}$ étant réels.

Une question importante est l'unergie absorbic par un système. On démontre que l'énergie absorbée a l'entré ou la sortie du quadripòle est respectivement :

$$
\mathrm{U}_{e} \cdot \overline{\mathrm{I}}_{e}=\overline{\mathrm{U}}_{\ell} \cdot \mathrm{l}_{c} \text { el } \quad \mathrm{U}_{s} \cdot \overline{\mathrm{I}}_{s}=\overline{\mathrm{U}}_{s} \cdot \mathrm{I}_{s}
$$

Le trait surligné signifie : nombre complexe conjugué $(j \rightarrow-j)$.

On démontre la proposition directement en partant de :

$$
u_{t}=\cos (\omega t-\hat{i}) \quad i_{c}=\cos (\omega t+\varphi)
$$

\section{RAPPEL DE QULLUUS PROPRIÉTES} DES OUADRIPOLES TINGMRES

(voir réfrences dités avant).

a) $a_{11}$ et $a_{2,2}$ :

Si on fait $I_{3}=0$ (par exemple : moteur électrique bloqué à la sortie de l'arbre ou conduite bouchée à une extrémité), le signe de l'énergie absorbée à l'entrée est $a_{1 \pi}$.

De mème, si on fait $\mathrm{I}_{e}=0$, le signe de l'énergie absorbée est $a_{22}$.

Si $a_{11}>0$, on dit que le quadripôle est passif à l'entrée lorsquil est en court-circuit à la sortie : il no pont alors qu'absorber de l'énergie.

De même pour $a_{\ldots . . .}$.

\section{b) Covplage interne des gudripótes.}

On appelle couplage le coefficient A douné par:

$$
A^{2}=\frac{A_{12}}{A_{21}}
$$

Si $A^{2}=1$, le quadripôle est représentable par un schéma électrique ne contenant que des résistances, selfs et capacités (qui peuvent néanmoins être négatives). On l'appelle quadripôle du genve «irchhoff 》.

Les quadripôles de Kirchhoff ont, en plus, les propriétés suivantes :

$$
a_{11}>0 ; \quad a_{22}>0 ; \quad a_{12}<a_{11}
$$

Ils sont toujours passifs, c'cst-à-dire ne peuvent qu'absorber de l'énergie en toutes circonstances. 
Si $A=0$ ou $\propto$, le quadripôle est dit « orienti ». Ces quadripôles ont les propriétés remarquables suivantes :

- La sortie ne réagit pas sur l'entrée;

- L'énergie à la sortie n'a rien à voir avec l'énergie à l'entrée, elle provient d'une source auxiliaire.

On retrouve done ici la notion de liaison sans réaction qui a servi à décomposer les installations de régulation au début de cet article.

\section{COUPLAGE DES QUADRIPÔYLES ENTRE EVXX}

On peut coupler les quadripôles de trois manières : en série - en parallèle - en chaîne (fig. 24).
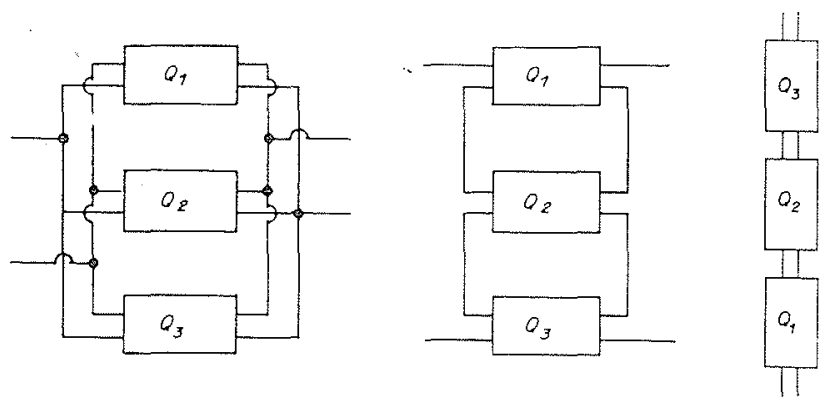

FIG. 24 .

On peut évidemment associer les divers modes de couplage. En général ", quand il reste quatre liaisons libres, l'ensemble forme eneore quadripôle. Si un ensemble de quadripôles contient un quadripòle orienté couplé en chaîne, l'ensemble est également un quadripôle orientí. Ces deux dernières propositions se démontrent directement par l'élimination des variables intermédiaires.

\section{III-5. - Etude des éléments des boucles de régulation.}

D'après ce qui ressort du paragraphe III-4, les éléments sont des quadripôles orientés; en effet. ils débutent par une liaison sans réaction.

Leurs relations fondamentales sont done, par exemple :

$$
\begin{gathered}
\mathrm{U}_{e}=\mathrm{A}_{11} \mathrm{I}_{e} \\
\mathrm{U}_{s}=\mathrm{A}_{21} \mathrm{I}_{e}+\mathrm{A}_{2.2} \mathrm{I}_{s} \\
\mathrm{~A}_{12}=0 \text { puisque : } \\
\mathrm{A}^{2}=-\frac{\mathrm{A}_{12}}{\mathrm{~A}_{21}}=0 \quad\left(\text { ou } \mathrm{A}_{21}=0 \text { si } \mathrm{A}^{2}=\infty\right)
\end{gathered}
$$

1. Nous ne ferons pas la discussion complete ici.
Couplons les éléments en chaine, comme cela est le cas dans les boucles de régulation (fig. 25):

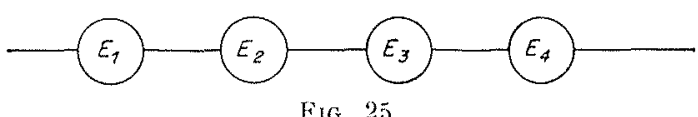

FIG. 25.

On a alors :

$$
\begin{aligned}
& \mathrm{U}_{e 1}=\mathrm{A}_{11} \mathrm{I}_{61} \\
& \mathrm{U}_{s 1}=\mathrm{A}_{21} \mathrm{I}_{e 1}+\mathrm{A}_{22} \mathrm{I}_{s 1} \\
& \mathrm{U}_{e 2}=\mathrm{B}_{11} \mathrm{I}_{e 2} \\
& \mathrm{U}_{x 2}=\mathrm{B}_{21} \mathrm{I}_{e 2}+\mathrm{B}_{22} \mathrm{I}_{s 2} \\
& \mathrm{U}_{e 3}=\mathrm{C}_{11} \mathrm{I}_{e 3} \\
& \mathrm{U}_{33}=\mathrm{C}_{21} \mathrm{I}_{e 3}+\mathrm{C}_{22} \mathrm{I}_{s 3}
\end{aligned}
$$

En plus :

$$
\begin{aligned}
& \mathrm{U}_{s 1}=\mathrm{U}_{e 2} ; \quad \mathrm{I}_{s 1}=\mathrm{I}_{e 2} \\
& \mathrm{U}_{s 2}=\mathrm{U}_{e 3} ; \quad \mathrm{I}_{s 2}=\mathrm{I}_{e 33}, \text { etc. }
\end{aligned}
$$

En éliminant, on obtient :

$$
\begin{aligned}
& U_{s 1}=\frac{A_{21}}{A_{11}}-\frac{1}{1-\frac{A_{22}}{B_{11}}} U_{01} \\
& U_{s 2}=\frac{B_{21}}{B_{11}}-\frac{1}{1-\frac{B_{22}}{C_{11}}} U_{e 2}
\end{aligned}
$$

Chaque iquation du type (2) caractérise un élément. Les éléments sont donc caractérisés par - une seule équation, tandis qu'un quadripôle est caractérisé par deux équations. C'est pour cette raison (qui découle des liaisons sans réaction) que seuls les éléments ont une individualité marquée.

$B_{11}$ est l'impédance d'entrée du premier quadripôle (celui qui est orienté) du deuxième élément. On peut considérer que $B_{11}$ fait partie de l'élément $\mathrm{E}_{1}$. L'élément est bien une «unité » puisque $B_{11}$ ne dépend pas des autres quadripôles de $\mathrm{E}_{2}$ (d'ailleurs très souvent $\mathrm{B}_{11}=0$ ) et on peut effectivement faire un « catalogue * des j̇éments, comme indiqué au $\mathrm{n}^{\circ}$ II-3.

On a alors :

$$
\begin{aligned}
& \mathrm{U}_{s ! 2}=\mathrm{F}_{2}(j \omega) \cdot \mathrm{U}_{e 2} \\
& \mathrm{U}_{s 1}=\mathrm{F}_{1}(j \omega) \cdot \mathrm{U}_{e 1}
\end{aligned}
$$

Chaque élément est caractérisé par une seule fonction $\mathrm{F}(j \omega)$. Les éléments sont bien plus simples à manier que les quadripôles.

On appelle $F(j \omega)$ la fonction de transfert de 
l'élément; elle le caractérise entièrement comme il ressort de tout ce qui a été dit jusqu'ici. Rappelons la signification de $\mathrm{F}(j \omega)$. Si on injecte un signal : $\mathrm{U}_{e}=\mathrm{A} \cos \omega t$ dans l'élément, il sort :

$$
\mathrm{U}_{s}=\mathrm{B} \cos (\omega t+\varphi)
$$

$\frac{B}{A}$ est la valeur absolue de $\mathrm{F}(j \omega)$; i est l'argument de $\mathrm{F}(j \omega)$. Si $\mathrm{U}_{c}=A \cos (\omega t+\psi)$, on peut représenter $\mathbf{U}_{e}$ par un vecteur dans le plan complexe, sa longueur est $\mathrm{A}$, son angle avec $\mathrm{O} x$ est $\psi$. $\mathbf{U}_{s}$ est alors le vecteur obtenu par la multiplication des nombres complexes $U_{e}$ et $F(j(\omega)$ ).

Quand $\omega$ varie, le point représentant $F(j \omega)$ dans le plan complexe $x o y$ varie : il décrit une courbe graduée en w. Cette courbe est la courbe de transfert (fig. 26).

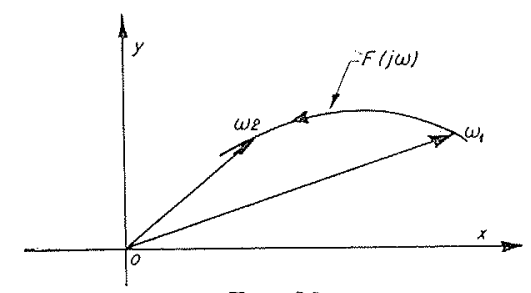

FIG. 26.

On lui associc une fleche montrant le sens de parcours quand w augmente.

Etudions maintenant te couplage en chaine des éćments linćaires.

De :

$$
\begin{aligned}
& \mathbf{U}_{s 1}=\mathbf{F}_{1}(j \omega) \mathbf{U}_{e 1} \\
& \mathbf{U}_{s 2}=\mathbf{F}_{2}(j \omega) \mathbf{U}_{e 2}
\end{aligned}
$$

et :

$$
\mathrm{U}_{1 s}=\mathrm{U}_{\mathrm{e}^{2} \mathrm{~s}}
$$

il résulte:

$$
\mathbf{U}_{s ! 2}=\mathbf{F}_{1}(j \omega) \cdot \mathbf{F}_{2}(j \omega) \mathbf{U}_{t 1}=\mathrm{F}(j \omega) \cdot \mathbf{U}_{a 1}
$$

La fonction de transfert $F(j \omega)$ est égale au produit $F_{1}(j \omega)$ par $F_{2}(j \omega)$. L'ordre n'intervient donc pas dans le couplage. La courbe de transfert résultant du couplage est obtenue par maltiplication de nombres complexes à partir des courbes de transfert élémentaires. On fait la contruction en général graphíquement. C'est ici l'origine de la méthode graphique (voir II-3).

\section{Exemples:}

1. Courbe de transfert d'Une condutte (linéarisée) EN PRENant EN CONSIDÉRATION Le SEUL COUP DE BŔLIER EN MASSE.
Equation non linéaire.

$$
\mathrm{H}-\frac{\mathrm{L}}{g \sigma} \frac{d \mathrm{Q}}{d t}-\mathrm{K}_{1} \mathrm{Q}_{2}=\frac{\mathrm{KQ}^{2}}{\mathrm{Y}^{2}}
$$

$\mathrm{H}$ : hauteur géométrique;

$\mathrm{L}$ : longueur de la conduite;

$g: 9,81 \mathrm{~m} / \mathrm{s}^{2}$;

$\sigma$ : section de la conduite;

$\mathrm{K}_{1}$ : coefficient de perte de charge de la conduite;

$\mathrm{K}$ : coefficient de débit de la vanne,

$\mathrm{Y}$ : ouverture de la vanne,

$q:$ débit.

Pour linéariser, on pose :

$$
\begin{aligned}
& \mathrm{Y}=\mathrm{Y}_{1}+y \\
& \mathrm{Q}=\mathrm{Q}_{1}+q
\end{aligned}
$$

D'où :

$$
\begin{gathered}
\mathrm{T} \frac{d q}{d t}+2 q=2 \beta \frac{\mathrm{Q}_{1}}{\mathrm{Y}_{1}} y \\
\mathrm{~T}=\frac{\mathrm{L}}{g \sigma} \frac{\mathrm{Q}_{1}}{\mathrm{H}}
\end{gathered}
$$

$\beta$ pourcentage de pertes de charge dans la vanne.

La fonction de transfert est donnée par :

$$
q_{0}=\frac{2 \beta Q_{1}}{Y_{1}} \frac{1}{2+j \omega \mathrm{T}} y_{0}{ }^{*}
$$

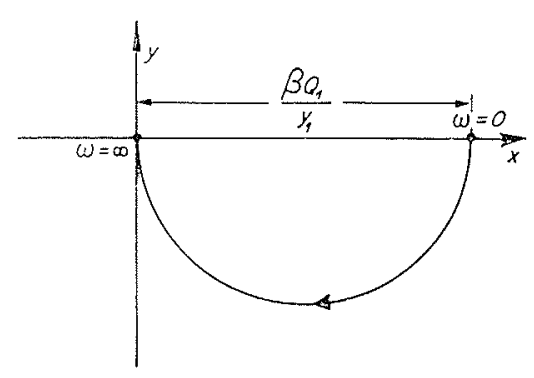

Fig. 27.

La courbe de transfert est un cercle :

2. Courbe de transfert' d'un régulateur a viTESSE PROPORTIONNELLE A L'ÉCART :

Equation :

$$
\frac{d y}{d t}=\mathrm{K}_{0}(\mathrm{~N}-m y)
$$

\footnotetext{
* Elle provient de $\mathrm{T} d q / d t+2 q=2 \beta \mathrm{Q} / \mathrm{Y}_{1} y$, où on a $\operatorname{posec}_{t}=q_{0} e^{e j t}, y=y_{0} e^{e j t}$.
} 
$K_{0}$ : rapidité de réponse;

$m$ : degré d'asservissement;

$\mathrm{N}$ : niveau dans le «bassin à niveau réglé »;

Y : ouverture.

Fonction de transfert :

$$
y_{0}=\frac{k_{0}}{j(1)+m} \mathrm{~N}_{0}
$$

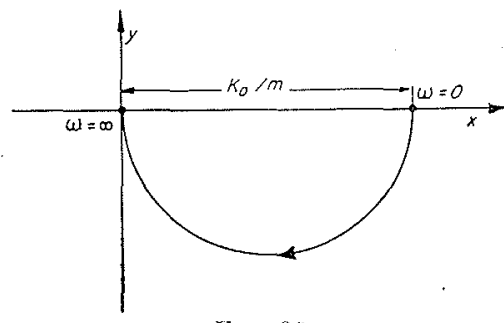

Fig. 28. cle.

La courbe de transfert est également un cer-

3. Couplage en chaine : condutte + RégulaTEUR, COURBE DE TRANSFERT, FIGURE 29.

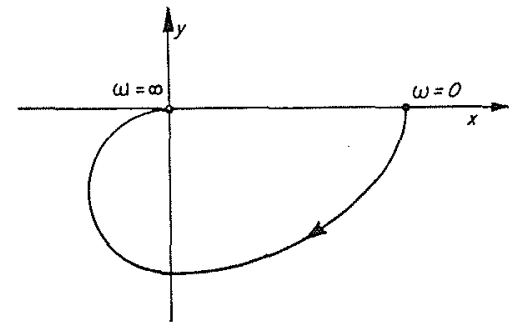

Fi(i. 29)

Nous nous sommes efforcés, jusqu'ici, de montrer toutes les particularités des boucles de régulation en nous tenant à des considérations élémentaires. Nous verrons le parti qu'on peut en tirer plus loin. Il reste néanmoins une dernière particularisation qui, quoique n'étant pas toujours applicable, est néanmoins souvent d'un secours trés puissant. Cette derniere particularisation ne se laisse malheureusement pas décrire d'une facon simple (du moins pas à notre connaissance). Il s'agit des relations entre l'amplification et le déphasage produit par chacun des éléments.

Reprenons l'analyse harmonique à ses débuts. Nous avions étudier la réponse d'un élément ou d'un quadripôle à une excitation $\mathrm{A} \cos (t) t$; nous allons maintenant étudier la réponse à :

$$
A e^{+\lambda t} \cos \omega t \text {. }
$$

Au lieu de :

$$
\text { A } e^{+\lambda t} \cos \omega t
$$

nous pouvons écrire :

$$
\mathrm{R}\left[\mathrm{A} e^{(\lambda+j \omega) t}\right] \text {. }
$$

$R(\ldots)$ veut toujours dire partie réelle de... On peut alors refaire toute la théorie en remplaçant $j \omega$ par $(\lambda+j \omega)$ [ou $p$, comme on écrit en calcul symbolique $(\lambda+j \omega=p)]$.

On aboutit a la fonction de transfert :

$$
F(p)=F(\lambda+j \omega)
$$

dont $\mathrm{F}(j \omega)$ est le cas particulice, $\lambda=0$.

Soit encore $x$ et $y$ les longueurs portées sur les axes du plan complexe dans lequel on trace les courbes de transfert $\mathrm{F}(j \omega)$ et $\mathrm{F}(\lambda+j \omega)$.

Alors à chaque valeur de $p=\lambda+j \omega$ correspond un vecteur, donc une valeur :

$$
z=x+j y
$$

et on a :

$$
z=x+j ! y=\mathrm{F}(\lambda+j(\omega) \quad \text { ou } \quad z=\mathrm{F}(p) .
$$

Les courbes $\lambda=\mathrm{C}^{\text {ste }}$ et $\omega=\mathrm{C}^{\text {ste }}$ sont orthogonales et définissent le champ à potentiel de la fonetion analytique $z=\mathbf{F}(p)$ ou de la fonction inverse $p=\Phi(z)$, telle que $\Phi[F(z)] \equiv z$.

Pour la suite, il nous faudra faire appel aux propriétés de fonctions analytiques. Nous supposerons connues un grand nombre de leurs propriétés. On pourra trouver celles-ci par exemple dans [61].

La fonction de transfert $\mathrm{F}(\lambda+j \omega)=\mathrm{F}(p)$ fait correspondre au nombre $p=\lambda+j \omega$ un nombre $z=x+j y$ :

$$
x+j y=\mathrm{F}(\lambda+j \omega) .
$$

On peut aussi en tirer :

$$
\lambda+j()=\Phi(x+j !))
$$

(T) étant la fonction inverse de F, \& permet de tracer dans un plan $\lambda$, (1) des courbes $x=C_{\text {sta }}$ et $y=\mathrm{C}^{\text {ste }}(\mathrm{fig} .30)$.

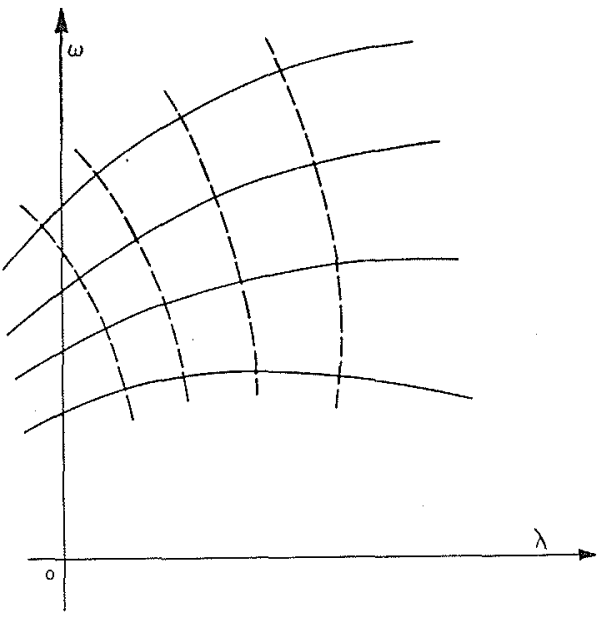

FIG. 30 
Ces courbes définissent la fonction analytique inverse : $p=\Phi(z)$. La connaissance de la courbe de transfert $\mathrm{F}(j \omega)$ dans le plan des $z$ donne les valeurs de $x$ et de $y$ sur le demi-axe positif du plan $p$. Les coefficients de la fonction $F(j \omega)$ (fonctions entières de $z$ et $e^{\sqrt{\varangle+\beta \tilde{\alpha}+\gamma^{2}}}$ dans la pratique) étant réels, "on connaît les valeurs de $x$ et $y$ pour les valeurs négatives de l'axe $o \omega$ par' symétrie. Or la connaissance des valeurs de $x$ ou de $y$ sur l'axe $o$ w et la connaissance des singularités de la fonction $F(p)$ dans un des demi-plans limité par l'axe $o \omega$ suffit pour déterminer $x$ et $y$ dans tout le plan. Nous voyons done que si on connaît les singularités dans un des demi-plans, la donnée de la courbe de transfert $F(j \omega)$ est surabondante. Or, les points singuliers sont très souvent des données physiques puisque ce sont eux qui «engeridrent » toute la fonction. C'est ceci qui est à la base de la simplification que nous allons décrire.

En réalité, ce ne sont pas les fonctions $x(\lambda ; \omega)$ et $y(\lambda ; \omega)$ qui sont les plus intéressantes, mais les fonctions $1 / 2 \log \left(x^{2}+y^{2}\right)$ et $\operatorname{Arc} \operatorname{tg} \frac{y}{x}$; c'est-à-dire logarithme de l'amplification et déphasage. Ces deux fonctions sont les parties réelles et imaginaires de $\log (x+j y)$.

Avant de passer au plan $(\lambda ; \omega)$, on fait done une transformation conforme du plan $x \circ y$ :

$$
Z=\log z
$$

Les courbes $\log A=C^{\text {stt }}$ et $\varphi=C^{\text {stt }}$ définissent done également une fonction analytique dans le plan $(\lambda ; \omega)$.

Si cette fonction n'a pas de singularité dans le demi-plan, à droite de l'axe $o(\omega$, on dit que l'élément caractérisé par cette équation est « à minimum de phase $»$. (On verra la raison de cette dénomination plus loin.)

Dans ce cas, il suffit donc de connaître $\log A$ en fonction de $\omega ;$; s'en déduit d'une façon unique.

Pour qu'un élément soit à minimum de phase, il faut donc que $F(p)$ ne devienne ni nul ni infini dans le demi-plan à gauche de $o w$. On a vu que les éléments ne contenant que des systèmes à constantes localisées avaient une fonction de transfert du type : fraction rationnelle de $\lambda+j \omega=p$. Dans ce cas, il suffit que cette fraction rationnelle n'ait pas de pôle ni de zéro à droite de l'axe $o \omega$. On verra qu'il n'y a, en général, pas de, pôle dans cette région.

Les éléments qui n'ont pas les propriétés requises pour être à minimum de phase s'appellent éléments « non à minimum de phase » (terme consacré par l'usage). D'une façon générale, on peut décomposer ces éléments en un élément à minimum de phase et un autre élément appelé « déphaseur pur».

Nous allons montrer cette décomposition sur un exemple :

Soit :

$$
F(p)=g(p) \cdot\left(p-p_{0}\right)
$$

$g(p)$ n'ayant pas zéro au pòle à droite de $o \omega$ et $p_{0}>o$.

Ơn écrit :

$$
\mathrm{F}(p)=g(p)\left(p+p_{0}\right) \times \frac{p-p_{0}}{p+p_{0}}
$$

On vérifie que l'amplification de $\frac{p-p_{0}}{p+p_{0}}$ est ćgale à 1, c'est pour cela qu'on appelle un tel élément un « déphaseur pur». En plus, ces éléments produisent toujours un déphasage négatif. C'est de cela que provient le nom : « à déphasage (négatif) ${ }^{1}$ minimum $\gg$ donné aux éléments ne contenant pas de déphaseur pur; dès gu'on ajoute un déphaseur pour constituer un ćlément non à minimum de phase, Ie déphasage (négatif) croît.

Pour plus de renseignements à ce sujet, on peut voir [9] [56].

Si on constitue un catalogue d'éléments, on peut décomposer une fois pour toutes chaque élément en élément à déphasage minimum et déphaseur pur. D'ailleurs, la plupart des éléments ne contiennent pas de déphaseur pur. D’après ce qui précède, il existe pour les éléments à minimum de phase une relalion entre le déphasage et l'amplification. Cette relation est:

$$
\Phi_{0}=\frac{\pi}{12}\left(\frac{d \log \mathrm{A}}{d \mu}\right)_{0}+\frac{1}{6 \pi} \int_{-\infty}^{+\infty}\left[\left(\frac{d \log \mathrm{A}}{d \mu}\right)-\left(\frac{d \log \mathrm{A}}{d \mu}\right)_{0}\right] \log \operatorname{coth} \frac{|\lg |}{2} d \mu
$$

avec :

$$
\mu=\log \frac{\omega}{\omega_{t}}
$$

(Voir [52] et $[56$ !

Le déphasage au point d'indice zéro dépend donc de toute la courbe d'amplification. $\frac{d \log \mathrm{A}}{d !}$ est la pente en $d b / o c t a v e ; ~ \Phi$ est en radians.

1. On verra que c'est le déphasage négatif qui est en général néfaste. 
En pratique, la fonction de pondération $\log \operatorname{coth}|\omega| / 2$ n'a une valeur non négligeable qu'au voisinage de $f=f_{0}$. Donc le déphasage en un point ne dépend pratiquement que de la pente de la courbe d'amplification en ce point, à une pente $n d b / o c t a v e$ correspond un déphasage de $-n \frac{\pi}{2}$, avec l'approximation $\operatorname{coth} \frac{|\mathrm{s}|}{2}=0$ pour $\rho \neq f_{0}$.

Pour les systèmes non à minimum de phase, il faut ajouter le déphasage produit par le déphaseur pur.

Rappelons qu'en régulation des turbines en rréquence-puissance, il existe toujours quelque part un déphaseur pur. Ceci est physiquement dù au fait que la puissance peut rester constante si le débit baisse (convenablement) et que la pression augmente dans la conduite forcée due au coup de bélier). On peut en général trouver une raison physique pour expliquer pourquoi tel ou tel élément est à déphasage minimum ou non.

Revenons maintenant aux éléments non linéaires. Pour les définir, il faut définir le quadripôle non linéaire orienté.

Ce dernier a une de ses deux relations fondamentales ne contenant que deux des quatre variables $\mathrm{U}$ et $i$.

On voit alors que l'élément non linéaire se définit exactement comme l'élément linéaire. Il est caractérisé par une relation non linéaire entre $\mathrm{U}_{6}$ et $\mathrm{U}_{*}$ et leurs dérivées et intégrales par rapport au temps :

$$
\Phi\left(\mathrm{U}_{\epsilon} ; \mathrm{U}_{*}\right)=0
$$

Cette relation définit entièrement l'élément.

On verra qu'en ce qui eoncerne la stabilité des boucles de régulation, l'élément est caractérisé par un ensemble de courbe de transfert. Pour l'instant, on ne peut pas aller plus loin que la relation (3).

\section{III-6. - Les comparateurs (ou discriminateurs).}

Pour terminer l'étude des régulateurs à boucles, il suffit maintenant d'étudier les comparateurs. Comme on l'a déjà vu, ils n'établissent pas, en général, la relation simple $q=q_{k}-q_{c}$, mais d'une manière générale :

$$
\mathrm{F}_{1}(j \omega) q=\mathrm{F}_{2}(j \omega) q_{s}+\mathrm{F}_{3}(j \omega) q_{c}
$$

On les décompose alors en trois éléments fictils ct un comparateur idéal (fig. 31) :

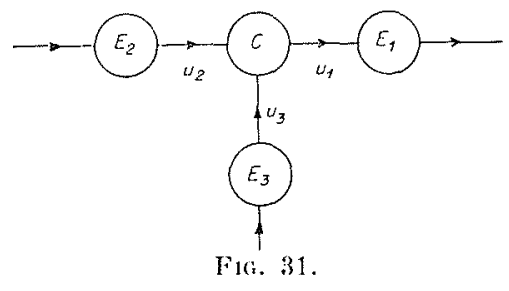

$$
\begin{aligned}
& \mathrm{E}_{3} \text { donne } u_{3}=-\mathrm{F}_{3}(j(1)) q_{3} \\
& \mathrm{E}_{2} \text { donne } u_{2}=\mathrm{F}_{2}(j(1)) q_{2} \\
& \mathrm{E}_{1} \text { donne } u_{1}=\mathrm{F}_{1}(j(1)) q \\
& \mathrm{C} \text { donne } u_{1}=u_{2}-u_{3}
\end{aligned}
$$

Les cléments fictifs ont toutes les propriétés des élément ordinaires.

Le mème processus est possible pour les comparateurs non linéaires.

On peut alors affirmer qu'un régulateur à boucles se compose uniquement d'éléments et do comparateurs idéaux.

Exemple: Un bassin donne la relation :

$$
\mathrm{s} \frac{d \mathrm{~N}}{d l}=q_{\mathrm{s}}-q_{\mathrm{r}}
$$

comme on a déjà vu.

On le décompose en :

$$
q=q_{s}-q_{0}
$$

et l'élóment E:

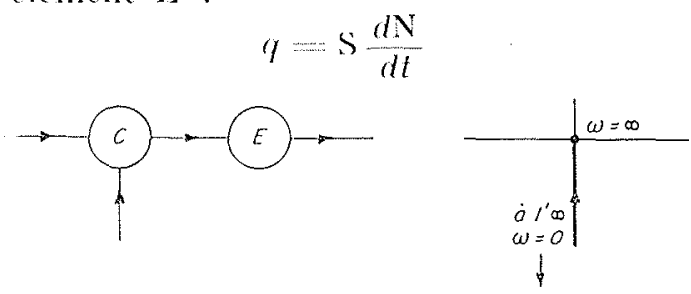

Fifi. 32.

La courbe de transfert de $E$ est une droite.

\section{IV. - THÉORIES ET MÉTHODES D'ÉTUDE DES INSTALLATIONS AUTOMATIQUES}

\section{IV-1. - Méthodes d'Hürwitz-Routh, Léonhard, etc.}

Les premieres méthodes appliquées aux régulateur's étaient valables pour tous les systemes physiques à constantes localisées.
On partait des équations du système et on déterminait ses frécuences propres : en particulier, on regardait si toutes ses fréquences étaient amorties ou non, d'où stabilité ou instabilité du systime.

Il est clair que la résolution d'un problème 
aussi général est plus compliquée que celle du frobleme très particulier posé par les régulateurs a boucles. C'est le srand mérite de Nyous'r d'avoir indiqué une méthode plus particulière englobant néanmoins à peu près tous les régulateurs à asservissements.

\section{IV-2. - Méthode de Nyquist.}

$$
[11],[34],[52],[54],[14],[48]
$$

La méthode Nyoust est basce sur la notion d'élément. Revenons à l'installation ayant une seule boucle. Elle se schématise par :

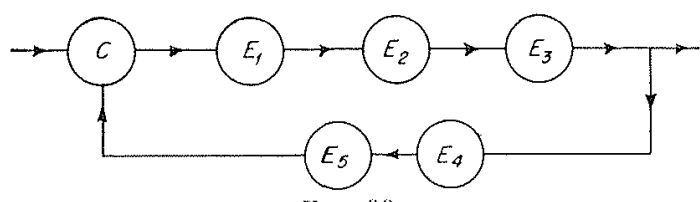

Fic. 333

ou $C$ est le comparateur, $E_{1}, E_{2}, E_{3}, E_{4}, E_{5}$, les déments.

Un ensemble d'éléments couplés en chaine ayant une fonction de transfert tout comme un scul élément, on peut aussi considérer le schéma suivant :

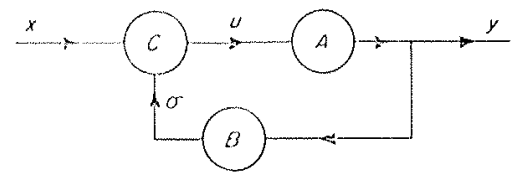

Fici. 34

A et $B$ sont des ensembles d'éléments ayant les fonctions de transfert $\mathrm{A}(j(1))$ et $\mathrm{B}(j(j))$.

L'orde domné à l'installation sera désigné par $\therefore$, l'exécution par $y$, la grandcur réglée par $u$.

Les equations reliant ces grandeurs sont :

$$
\begin{aligned}
& u=x-\sigma \\
& y=\mathrm{A}(j \omega) \imath \\
& \sigma=\mathrm{B}(j \omega) y
\end{aligned}
$$

I'où :

$$
y=x \frac{\mathrm{A}(j \omega)}{1+\mathrm{A}(j \omega) \mathrm{B}(j \omega)}
$$

La valeur réglée $u$ est :

$$
u=\frac{x}{1+\mathrm{A}(j \omega) \mathrm{B}(j \omega)}
$$

Pour que l'exécution $y$ soit égale à l'ordre $x$ ou fue le parametre réglé soit constant, il faut que :

$$
A(j(1))=1+\mathrm{A}(j(1) \mathrm{B}(j(1)) .
$$

En hydraulique, on a presque toujours $B(j(1))=1$, il ne reste done que la condition $A(j \omega)=\infty$.

Le régulateur exécute d'autant mieux l'ordre que celte condition est vérifiée par un plus grand nombre de valeurs w.

En praticue, on réalise $A(j(1))=\infty$ toujours pour $(\omega) \rightarrow 0$, et ceci au moyen d'un intégrateur. En effet, si $\mathrm{U}_{s}=\int \mathrm{U}_{t} d t$, on a, en $j(1)$ :

$$
U_{s}=\frac{U}{j(1)} \quad \text { ol } \quad F(j(1))=\frac{1}{j(1)}
$$

Done $\mathrm{F}(j \omega) \rightarrow \infty$ quand $\omega \rightarrow 0$. Celte condition garantit un niveat moyen correct de la valeur à régler.

On voit aussi pourquoi les installations à régulation automatic|ue el les systemes de commande indirecte asservis sont très voisins. En cflet, $x=y$ est ćquivalent à $u=0$.

Domnons quelques exemples d'installations aumatiques : on va regarder quelles sont les significations des symboles $x, u, y, \mathrm{~A}, \mathrm{C}$, et de l'intégrateur dans quelques cas industriels comme la régulation des turbines ou la régulation d'un niveau constant en irrigation.

\section{Régulation des turbines}

$x=$ puissance demandée par le réseau $\mathrm{P}_{u}$,

$y=$ puissance $\overline{\mathrm{Q}} \mathrm{QH}$ fournie par la retenue à l'installation $\mathrm{P}_{a}$,

$u=$ différence des puissances $x$ et $y$.

La vitesse de rotation of du groupe est liée à $l l$ par :

I $\frac{d \Omega}{d t}=\iota$ (I moment d'inertie du sroupe)

Si :

$$
u=0 \quad \Omega=\mathrm{C}^{\mathrm{st}} \mathrm{u}
$$

Comparateur C : les pieces tournantes; elles donnent :

$$
\mathrm{I} \frac{d t}{d Q}=\mathrm{P}{ }-\mathrm{P}_{u}=u
$$

De mème que le bassin examiné dans un exemple antérieur, les pièces tournantes jouent à la fois un rôle de comparateur et d'intégrateur :

$$
\Omega=\frac{1}{\mathrm{I}} \int_{0}^{t} u d t
$$




\section{Réglage d'un niveau dans un bassin}

$x$ : débit demandé par le réseau d'adduction d'eau,

$y$ : débit demandé à la retenue,

$u$ : différence des débits $x$ et $y$.

Le niveau réglé $N$ est lié à $u$ par :

$$
\frac{d \mathrm{~N}}{d t}=u
$$

$\mathrm{Si}$ :

$$
u=0 \quad \mathrm{~N}=\mathrm{C}^{\mathrm{st} t}
$$

C: le bassin (il est également intégrateur).

\section{Appareil de mesure à asservissement}

$x$ : valeur de la grandeur à mesurer,

$y$ : valeur de la grandeur indiquée sur le cadran de l'appareil,

\section{$u$ : erreur :}

C: le comparateur est, en général, un pont de Wheatstone dans les appareils électriques ou électroniques.

$B$ peut exister dans ce cas.

Pour perfectionner la boucle de régulation, on pourrait s'aranger pour que $A(j \omega)=\infty$ pour de nombreuses valeurs de $\omega$. En réalité, ceci ne serait pas prudent pour un appareil mécanique ou hydraulique non destiné au laboratoire. En effet, $\mathrm{A} j(\omega)=\infty$ veut dire amplification infinie; les boucles contenant des amplifications trop grandes deviennent facilement instables, comme on verra plus loin.

\section{Stabilité}

Une boucle est stable si, à un ordre $x$ très petit, correspond une exécution $y$ très petite aussi.

Si $1+\mathrm{A}(j \omega) \mathrm{B}(j \omega)=0$, à $x=\varepsilon$ correspond $y=\infty$ : l'installation est instable.

Regardons de plus près la condition :

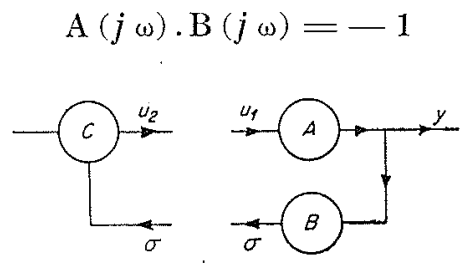

FIg. 35 .
Le produit $A(j \omega) . \mathrm{B}(j \omega)$ est la fonction de transfert de la boucle ouverte sans comparateur ${ }^{*}$.

En effet, admettons que $u_{1}=A \cos \omega t$; dans ce cas, on tire de :

$$
\begin{aligned}
& \mathrm{A}(j \omega) . \mathrm{B}(j \omega)=-1: \\
& \sigma=-\mathrm{A} \cos \omega t ; \sigma=-u_{1} .
\end{aligned}
$$

Or, le comparateur donne $u_{2}=-\sigma$; donc, dans ce cas, $u_{1}=u_{2}$ : si $\mathrm{A}(j \omega)$. B $(j \omega)=-1$, l'excitation $u_{1}=A \cos \omega t$ produit une réponse $u_{2}=u_{1}$ et lorsqu'on reforme la chaine, elle est susceptible d'osciller avec la fréquence $\omega$.

Traçons (fig. 36) la courbe de transfert de la chaine ouverte :

$$
\mathrm{F}(j \omega)=A(j \omega) \mathrm{B}(j \omega) .
$$

Si elle passe par - 1, Ja stabilité est juste limite. A $\cos \omega t$ n'est ni amorti ni amplifié, mais juste entretenu quand la chaîne est fermée.

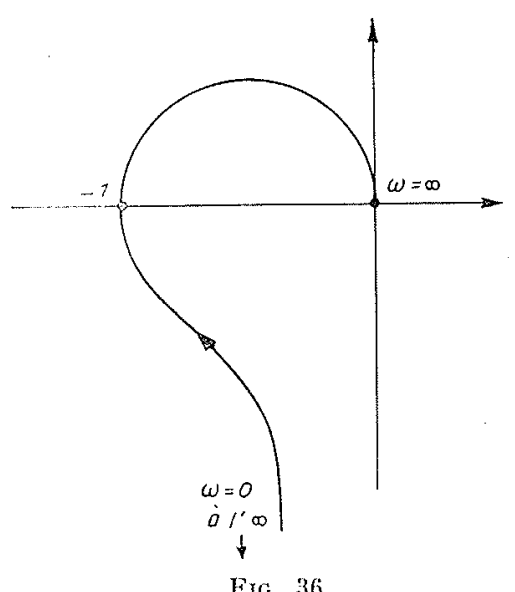

Si la courbe passe près du point - 1, l'oscillation $\mathrm{A} \cos \omega t$ est légèrement amortie ou légèrement amplifiée dans la chaine fermée ${ }^{2}$. On voit pourquoi le point - 1 joue un rôle essentiel.

Nyouist a montré que l'installation est stable si la courbe de transfert de la chaîne ouverte décrite de $\omega=0$ vers $\omega=\infty$ laisse le point -1 à sa gauche.

Les mathématiciens ont vivement critiqué le critère de Nyourst. Effectivement, il convient de le compléter en disant : l'installation est stable, si le critère de Nyouist est satisfait et si la chaîne ouverte est stable elle aussi, c'est-à-dire si :

$$
\mathrm{A}(j \omega) \neq \infty \text { pour } \omega \neq 0 \text {. }
$$

\footnotetext{
* On pourrait prendre + 1 comme point critique, mais on verra par la suite qu'il est préférable de prendre -1 .

2. $F(j \omega)=A(j \omega)$. B. $(j \omega)$ est la fonction de transfert de la chaîne ouverte, mais la stabilité considérée ici est une propriété de la chaîne formée.
} 
On a déjà vu que $A(j \omega)=\infty$ améliorait la précision, mais en pratique on s'arrange pour que $\mathrm{A}(j \omega) \neq \infty$ pour $\omega \neq 0$.

En cffel, une ouverture aceidentelle de la boucle (bouchage do circuit d'huile d'un régulateur, etc.) pourrait provoquer un pompage détériorant l'installation.

Par ailleurs, on montre physiquement que pour les éléments $A(j \omega)$ est toujours différent de linfini. Seule l'existence de boucles secondaires insLables pourrait donner $A(j(0)=\infty$ ceci tant (Iu'on se limite au domaine linéaire).

S'il y a $n$ valeurs (1) telles que $\mathrm{A}(j(1))=\infty$, le critère de Nyouste devient : la chaine fermée est stable si la courbe de transfert de la chaine ouverte tourne $n$ fois autour du point - $1:$. Co point se trouvant à gauche de la combe décrite dans le sens $\omega=0$ à $\omega=\infty$.

\section{Définition de la slabilite absolue}

On dit qu'une installation est absolument stable si les points de la courbe de transfert correspondanle silués sur l'axe réel sont tous à droite du point - 1 .

En effet, les vieillissements et les accidents produisent a peu prés toujours des diminutions d'amplitude dans la chaine fermée.

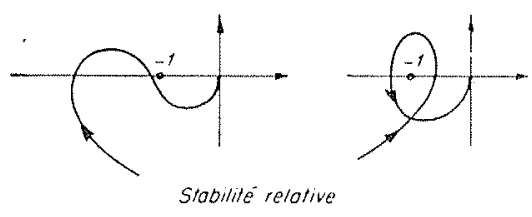

Fik. 37

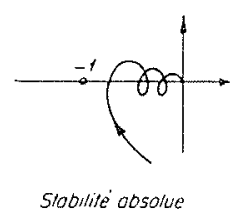

Les installations à stabilité relative sont stables mais dangereuses pour l'avenir.

Le diagramme de NYourst peut aussi servir à déterminer la fréquence dont l'amortissement est te plus petit et cet amortissement : $\omega_{0}$ et $\lambda_{0}[297$. Cette détermination se déduit tout de suite de l'analyse harmonique généralisée décrite à propos des éléments à minimum de phase. On injecto $\mathrm{A} e^{\lambda t} \cos \omega t$. Si $\mathrm{A}(\lambda+j \omega) \cdot \mathrm{B}(\lambda+j \omega)=-1$, $\lambda$ et w définissent un mouvement possible.

On a va que les courbes $\lambda=C^{\text {ste }}$ et $\omega=C_{\text {ste }}$ sont orthogonales et constituent les lignes d'un champ à potentiel. Connaissant une des lignes $\lambda=$ Cste du champ (par exemple $: \lambda=0$ ) graduée en w, on peut trouver l'ensemble des lignes du champ.

Cette détermination peut se faire graphiquement par la méthode dite des «petits carreaux».

3. Il faut compter la valeur $\omega=\omega_{1}$ pour \& $/$ » valeurs si $A\left(j \omega_{1}\right)$ devient o d'ordre $k$.
Connaissant ce champ, partout on peut résoudre l'équation :

$$
\mathrm{F}(\lambda+j \omega) \equiv \mathbf{F}(p)=-1
$$

donc les solutions $\lambda$ et $(1)$ sont des fréquences propres de la chaine fermée.

En effet, la chaine fermée oscille si :

$$
\mathrm{F}(\lambda+j \omega)+1=0,
$$

comme on a vu plus haut.

D'ailleurs, l'existence de la fonction analytique $\mathbf{F}(\lambda+j \omega)$ permet de démontrer rigoureusement te critere de Nroust. Ici nous voulons simplement mentionner que la construction graphique par « petits carreaux » qui permet d'obtenir un des couples de valeurs $\lambda$ et $\omega$ correspondant au point - 1 . On démontre que e'est en sinéal le couple de valeur ayant entre tous la valeur la plus petits de $\lambda$, donc correspondant à l'oscillation la moins amortie.

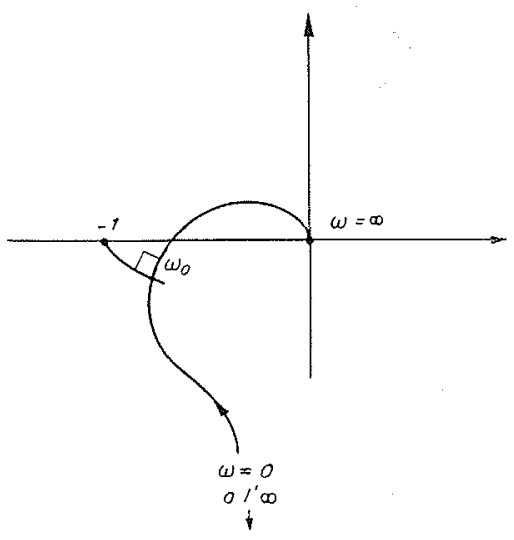

FIG, 38 .

En pratique, il suffit d'abaisser une « normale courbe $\gg$ du point -1 à la courbe $F(j \omega)$.

En général, on ne peut pas abaisser facilement plusieurs «normale courbe» du point -1 à la

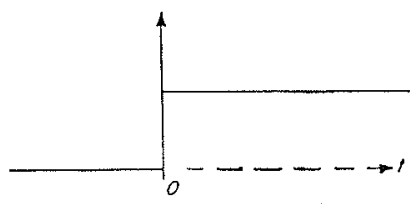

FIG. 39.

courbe de transfert. Cela signifie que les autres oscillations propres sont nettement plus amorties que la première. Une perturbation du genre échelon unité (fig. 39) donne alors naissance à des oscillations très rapidement voisines de :

$$
A e^{+\lambda a t} \cos \left(\omega_{0} t+\varphi\right) \text {. }
$$


$\lambda_{0}$ ou $\frac{\lambda_{0}}{\omega_{0}}$ caractérise donc bien l'amortissemcnt des oscillations de la chaîne.

\section{Retout aux colubes de Weygel}

Si on a pour les différentes régions du plan fréquence amplitude ( $n^{\circ}$ II-3, fig. 5) différentes courbes de transfert, on les limite aux fréquences pour lesquelles elles sont acceptables et on trace les différentes normales courbes du point -1 .

\section{Exemple :}

$\mathrm{C}_{1}$ est valable pour (1) compris entre 0 et $\left(_{1}\right)_{1}$.

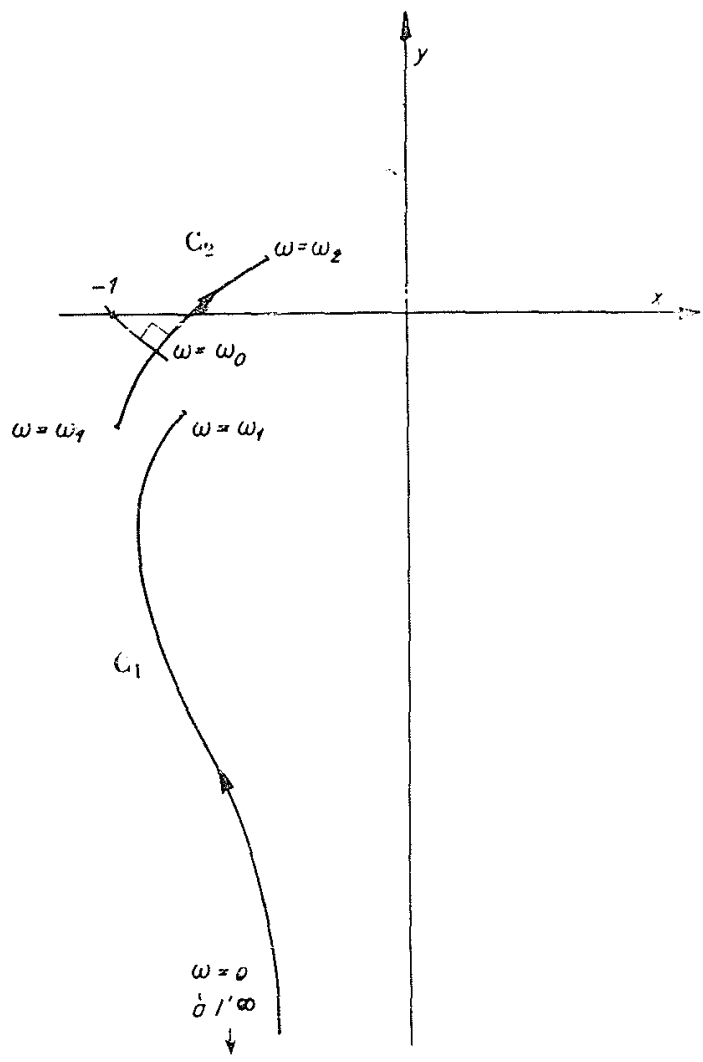

Fig. 40.

$C_{2}$ pour $\omega$ compris entre $\omega_{1}$ et $\omega_{2}$.

Si $\omega>\omega_{2}$, le système ne laisse plus rien passer. On en deduit $\omega_{0}{ }^{*}$.

\section{IV-3. - Systèmes à minimum de phase.}

Nous avons vu que pour les systèmes à minimum de phase, la donnée de la courbe de trans-

* Théoriquement, cette méthode est sujette à caution. Pratiquement, elle marche toujours. fert était surabondante; il suffit de se donner $\log A$ en fonction de $\log f$. Ceci est très important puisçu'il suffit de s'oceuper de deux variables au lieu de trois.

On verra dans le paragraphe suivant que le déphasage est pratiquement toujours négatif. Il en résulte que la courbe $\log \mathrm{A}$ en fonction de $\log f$ est toujours descendante. (D'après ce qu'on a vu au paragraphe III-5.) Comme, en plus, A est en général infini pour $\omega=0$ (d'après ce qu'on a vi à propos de la précision), la courbe $\log \mathrm{A} / \log f$ coupe l'axe $\log A=0$, en un seul point, c'est-àdire en un point et un seul de l'axe of, on a : $\mathrm{A}=1$; soit ce point : $f=f_{1}$.

On appelle cette fréquence $\hat{f}_{1}$ : fréquence de coupure. La figure 41 montre sa position sur le diagramme de NYQuist :

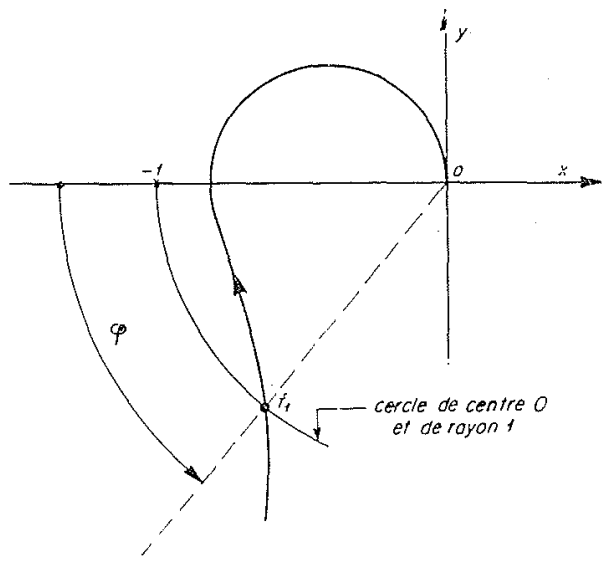

FIG. 41.

L'angle $\varphi$ s'appelle marge de phase : il est compté à partir de l'axe négatif ox. Pour que le système soit stable, il faut donc que $0>0$. D'après ce cqu'on a va à propos des systèmes à minimum de phase, il faut pour ceci que :

$$
\frac{d(\log \mathrm{A})}{d u}<12 d b / \text { octave. }
$$

pour la fréquence de coupure $f_{1}$.

Il suffit done, pour ces systèmes, de tracer $\log \mathrm{A}$ en fonction de $\log f$ et de vérifier que $\frac{d \log \mathrm{A}}{d \log I}<12 \mathrm{db} /$ octave pour la fréquence telle que $\log A=0$ pour être sûr de leur stabilité.

Si le système contient un déphascur pur, il f'aut calculer son déphasage $\Psi$ pour $f=f_{1}$ et vé. rifier la relation $\frac{d \log \mathrm{A}}{d \log f}<12\left(1-\frac{\mathrm{T}}{\pi}\right) d b /$ octave; $\Psi$ en radians.

Pour les éléments couplés en chaine, les diagrammes $\log A / \log f$ se combinent facilement par simple addition : $\log \mathrm{A}=\log \mathrm{A}_{1}+\log \mathrm{A}_{2}$. 
La recherche de la stabilité est done trés simple pour les systimes a minimum de phase of mène pour ceux qui contiennent des déphascurs purs simples.

\section{V-4. - Les installations automatiques en tant qu'ñtégrateurs.}

Nous avons décrit, jusqu'à présent, les régulateurs en mettant en valeur toutes les particularités qui permettent d'employer rationnellement la méthode de Nrouster pour les installations linéares.

En réalite, les installations de régulation ont cneore une particularite importante. Elle nous servira pour étudier les installations non linéaires. Cette particularité est la suivante : la tres srande majorité des déments déphasent en arriere. Langle de $F(j \omega)$ est alors toujours négatif. Ainsi les intégrateurs, les inerties, les régulalours à vitese proportionnelle à l'écart, elc...

Chaque déphasage de $\frac{\pi}{2}$ en arricre correspond à peu près à une intégration. Le point - 1 correspond a deux intégrations.

\section{(A suivre.)}

\section{Bibliographie}

1 - Acmúras : Influence de l'inertie de l'anu sur la stabilité d'un groupe hydro-électrique.

Honille blanche, nov, 1945, p. 81 (stite, mats 1946).

2 - Alméras : Stabilité des groupes hydro-éledriques. Hontlle blanche, nov.-dec. 1946, p. 407 .

3 - Amónas : Caracteristiques des resulatones. Honille Blanche, no $6 / 1947$, nov. -déc.

4 Amiras : Procedes d'amélioration des qualites de reglage des groupes hydro-electriques. Hoitlle Blanche, no $1 / 1049$, p. 38 .

5 - Armeras : Cours de regulation.

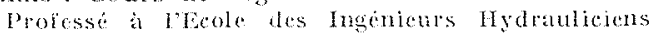
de Cirenoble.

(i)- Ancor: Complements de mathematicues. Entions de la Rever doplique, 1949.

7 -... Arpert : Traté de mécanique rationnelle, vol. If, p. 395.

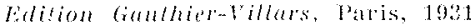

8-Baran : Cours deatectricite theorique.

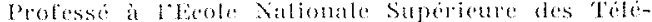
communteations, tomes I, Ty:3.

9) B..- Barard : Relation entere les pallies reelles of imaginaires des impedances ol delemination des impedances en fondion de lane des parties. R.G.E.. mai 1435, b. 609

10 - Bror el Vox Kanmas : Les méthodes mallématiques de l'ingénieur-éditeur.

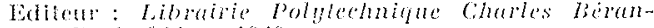
yer, Patismliege, 19:19.

11 - Bons: Notwork amalysis and foclback amplidien design.

Lititeul : Yan Noshand, Xew-York, 1945.

12 - L. BOÉ : Dipôles at quadripôles. Edition : L. Chirtm, Pallis.

13 - Borminox : Quelques theoromes sur les oscillations propres des systèmes de circuits. R.G.E., mat 1023, D. Gati.

14 - Brown et Canprert : Principtes of Servomed: anisms.

Editeur : T. Whly and Sons, Ine, New-rork, t918.

15 - Canen : Nouvelles analogies electromécandques. Bulletin S.F.E., 1950, P. 2\$8.

16 - CAHN et Desmis : Analysis of some hydratic components used in regulators and servomechanisms.

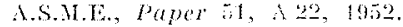

17 - Covprigna : Sur les conditions de stabilite des systemes oscillants.

C.R.A.S., p. 59.t, sem. 1943
18 - Cownon : Etude des proprictés d'un réglage automatique.

Rulletin leshnique ate la Suisse Romande, avlil 19) $17,1^{*} 4$, p. 10\%.

19)-Cukvon: Influence des phénomènes de coups te bélier sur te reglage de la vilesse des lurbines hydrauliques.

thotille Blanche, mars-anvil 1949, p. 163.

20 - Covsom: Contribution à l'etude d'un réglage automatique.

Bulletin Associulion Snisse des Electriciens, no 18. 1950, p. 673.

21 - Daxen : Accélération du réglage de la vitesse des turbines hydrauliques. These de doetorat, publies dans la Honille Blanche. $11^{\circ} 1-2,1918$.

22 - Danar : Le statisme des régulateurs et son élimination. Honille Blanche. $1101 / 1950$, p. 15.

23 -... Daver : Lévolution des métrodes de répatition de la charge daus les rescaux intereonnectés. R.G.E. o(tob) 1916 , p. 103.

24 - Droox : Considerations sur les regulateurs des groupes gemerateurs hydro-ilectriques de basse chute. R.C.... arout 1918, p. 315.

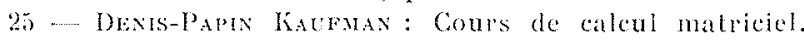
Edition: Albin Wichel, Paris, 1951.

26 -- Destouches: Principes fondamentaux de physique therique, tome II. bifition : Hermann at Cie, Paris, 1912.

27 - Dethn : Thérie des serromicanismes at relais. Onde Electrique octobre 1950, p. 438.

28 -.- Favne: Sur la genélalisation doune analogie chlo cinq phénomenes de la mécanique. linlefin lechnique de la Suisse Romande. 11/2. noy at 9 dec. 1944 .

29) - Gabex : Etude de la stabilite d'un réglage automatique de vilesse par des diagrammes vectoriels on vue de lutilisation du critere de Nyquist. hajormations techniques charmilles, $10^{\circ} 2, \quad$ p. 1 (aussi (a livre)

30 -..-Gabex: Asservissement fomporaire el acederomètre.

Iinlletin technique de la Suisse romande, $13 \mathrm{et}$ 27 mats 1988 , pp. 61 et 77 . - Aussi en livre, editeur : E. Ronffe el Cie, Lausanne, Suisse.

31 - Gabr: Influence de certaines caracteristiques intervenant dans la condition de stabilité. La Honlle Blanche, $\mathrm{n}^{\circ} 3 / 1948$, mai-juin. - Lègerement modifié dans un live. Editeur: Edilions la Concorde, Lausanne, Suisse, 1949. 
32 - Ganen : Considérations sur le probleme de stabilité.

Editions: La concorde. Lausanne, Suisse.

33 - Hermanx et Souran : Un cpitère de stabilité pout les équations caractéristiques à coefficients récls ou complexes.

La Recherche Aesonaudique, mai-juin 1949, p. 19.

34 - Janes, Nichols et Philipes : Theory of Servomechanisms.

Editeur : McGraw Hill Bool: Cy., Inc., New-York, 1947 .

35 - Kieser : Die Reglung der Wasserturbinen. Eloktrotechniti and Maschienenball, $1^{\circ} \quad 15 / 16$, 14 avil 1944, D. 180 .

36 - Lochenutuger : Analysis contactor servomedanisms. Elocirical Enginecring, arril 1950.

37 - G. Kron : Tensorial analysis of control systems. fournal of applied mechanies, june 1948, p. 107.

38 - Kontzuane, Danim, Ma Man Yuan : Stabilite de systèmes de réglage R.G.F.e, mar's 1952 , p. 142. R.G.E. mars $195^{2}$, p. 149.

39 - Le Branc: La régulation automatique de hatute précision. Mesure, annee 19.15, p. 429 .

40 - Lemmans: Note sur la précision maximum des servomécanismes parfaitement stables. Onde electrique, juin 1950, p. 267.

41 LehmanN : Progrès récents dans la conception des servomécanismes.

Office National de Recherehes Aeronatirues, ONERA, vol. 2, pp. 1 à is, aốt 1947 .

42 - Léonhand : Die Selbstätige Reglung. Editeur : Springer Verlag, Berlin, 1949.

43 - Loen et CAHEN : Des réseaux électriques aux transmissions mécaniques. Inulletin de l'Association Technique Maritime el Aéronantique. $\mathbf{1 9} 48$, p. 107.

44 - Lutri : Reglerschwingungen und schiefwinkelige Vektordiagramme. Sehveizertsche Butzeitung, avil 19\$2, p. 171.

45 - Lutri : Conditions d'amortissement des équations de régulateurs d'un ordre queleonque. Escher-Wyss - Mitteillungen, 15/16, 1942-1943, 1). 90 .

16 - Ma Man Yuan et Compon : Sur les criteres de stabilité de Routh et de Hurwitz. bulletin des Sciences mathématiques, fome LXXI, année 1948. t.7 -... Ma Mn YUas: Communication a l'A.E.R.A. Mai 1950

48 - Mac Corr : Fundamental theory of̈ servomechanisms.

Editeur : ran Vostrand, Vew-York, U.S.A.

19) - Mac Mulan: Theory of control. Editeur : th the Cniversily Press Cambridge, $195 \mathrm{j}$.

50 -- Maga: Indrekte Regulierung der Türbinen mit Benicksichtigung der Massenträgheit des Zullusswassers.

Zeilschift fï das fesamle Türbinenuesen, 1910, 1. 273.

51 - Mronsky : Non linear mechanies.

Edition : Edwed brolhers, Ine, Arm Harbor AI chigat1, 197\%, U.S.A.

52 -... Nasho : Les systemes asservis. Edition de la Reme doplatue, 1951.

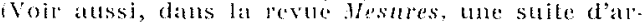
tickes commeneant dans fe munero de mars 1951 1). 91.

53 -... Nasse : Etude de la stabilite du reglage de vitesse d'un groupe hychro-électrique. Rulletin de la S.F.E. janvick 1951, p. 9.

54 - W. W Oprete : Crundgesetze der Regelung.

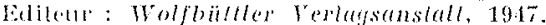

5j- - Panmexten : Analogies entre systemes électriques ef mécaniques.

Revme S.W., no 2, anil 1949 , p. 9.

56 -... F.-H. Raymoxb : Transformed de Hibert et Relatlions de Bayared-Bode.

thates des Tetecommunications. oct. 1951, p. 262.

57 -... F.-H. Rarnoxd : Electronique et Automatisme.

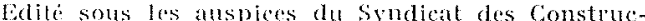
teurs francais des Machines-Outils 2 bis, rue de la Batume, Parisi.

58 -- Sernentian : Stabilité de la regulation des turbines lyydrauliques.

linlletin Escher-Wyss, tome 21/22, 1948-1949, p. 43.

59 - SToker : Non linear vibralions. Interscince Publishers, Ine, New-Xork, 1950.

(6) - Thmoner : Response of Physical systems. Edileul : J. Wiley and Sons, lue, New-York, 1950.

61 - Vamoos : Cours d'analyse mathematique, tome I: Theorie des fonctions. Editeur : Itesson el cie, Paris, 19.2

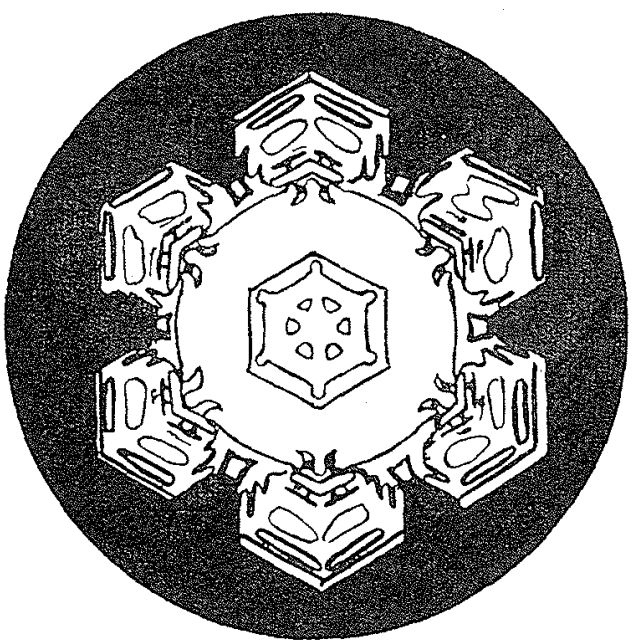

